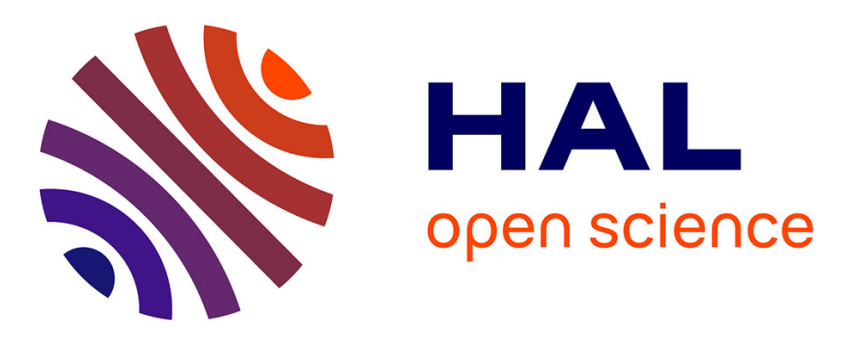

\title{
Numerical modelling of boom and oil spill with SPH
}

Damien Violeau, Clément Buvat, Kamel Abed-Meraïm, Emmanuel de Nanteuil

\section{To cite this version:}

Damien Violeau, Clément Buvat, Kamel Abed-Meraïm, Emmanuel de Nanteuil. Numerical modelling of boom and oil spill with SPH. Coastal Engineering, 2007, 54 (12), pp.895-913. hal-00375367

\section{HAL Id: hal-00375367 https://hal.science/hal-00375367}

Submitted on 15 Apr 2009

HAL is a multi-disciplinary open access archive for the deposit and dissemination of scientific research documents, whether they are published or not. The documents may come from teaching and research institutions in France or abroad, or from public or private research centers.
L'archive ouverte pluridisciplinaire HAL, est destinée au dépôt et à la diffusion de documents scientifiques de niveau recherche, publiés ou non, émanant des établissements d'enseignement et de recherche français ou étrangers, des laboratoires publics ou privés. 


\title{
Numerical Modelling of Boom and Oil Spill with SPH
}

\author{
D. Violeau ${ }^{\mathrm{a}, *}$, C. Buvat ${ }^{\mathrm{a}}$, K. Abed-Meraim ${ }^{\mathrm{b}}$ and E. de Nanteuil ${ }^{\mathrm{c}}$ \\ ${ }^{a} E D F R \& D$ / Laboratoire National d'Hydraulique et Environnement, 6 quai Watier, 78400 Chatou, France \\ ${ }^{\mathrm{b}}$ University of La Rochelle / LEPTAB, Pôle sciences et technologie, avenue Michel Crépeau, \\ 17042 La Rochelle cedex 01, France \\ ${ }^{\mathrm{c}}$ CEDRE, 715 rue Alain Colas, CS 41 836, 29218 Brest cedex 2, France
}

\begin{abstract}
The protection of coastal areas against oil pollution is often addressed with the use of floating booms. These bodies are subject to a design always based on physical models. Indeed, the numerical modelling of a two-phase flow (oil and water) with complicated free surface in the vicinity of a floating body is a challenging issue. The Smoothed Particle Hydrodynamics (SPH) Lagrangian numerical method is appropriate to such simulations since it allows the modelling of complex motions and fluid-structure interactions. In this paper we first study the mechanism of oil leakage by entrainment due to combined turbulent production and buoyancy. Then, we present the main features of the SPH method in a turbulent formalism and apply this model to predict the motion of a boom and an oil spill in an open-channel and a wave flume, for three types of oil (heavy, light and emulsion). Results are compared to experiments and used to depict criteria for oil leakage. It appears that oil leakage by entrainment occurs when the surface water velocity upstream the boom exceeds a critical value which was estimated as $0.5 \mathrm{~m} / \mathrm{s}$ for a light oil under steady current. A more accurate criterion is derived from theoretical considerations and compared to numerical experiments. An extensive use of this model should extend our knowledge regarding the mechanisms of oil leakage under a boom and allow a better an easier design of booms in the near future.
\end{abstract}

KEY WORDS: SPH, boom, oil spill.

\footnotetext{
* Corresponding author. Tel.: +33(0)1 30877831

Fax: +33 (0)130878086

E-mail address: damien.violeau@edf.fr
} 


\section{INTRODUCTION}

Floating booms are, if not the main, one of the most widely spread equipment used to respond to an oil spill in marine or inland waters (figure 1). Being based upon the principle of containing a floating pollution at the sea surface, they are commonly used for various purposes, either as protection tools (by preventing the oil to enter and impact specific sites), or to assist the recovery of this oil at sea, being towed around the slicks in order to confine and thicken them before they are collected by specific floating skimmers and pumps. The diverse types of existing floating booms are very numerous, and adapted to various uses and situations, from calm inland waters to open sea. However, all of them are generally composed of the same main five components: a float and a ballasting device to ensure the buoyancy and floating behaviour, a freeboard and an immerged skirt to effectively confine the pollution, and a tension member to absorb the stresses.

Despite their being widely used, floating booms are, in practical, far away from being fully efficient, and feature multiple failures. The need for a better understanding of these failures, and, thus, for quantifying their limits and defining their conditions of use and possible improvements, has let for a long time scientists study floating booms from a theoretical point of view. Such studies always revealed difficult problems to solve, regarding the complexity of the situation to model. Modelling such a complex behaviour is also a numerical challenge, due to the numerous difficulties occurring in such circumstances: a two-phase turbulent flow with fluid-structure interactions. Traditional method like finite element or finite volume may fail in simulating such a complexity, except through the VOF point of view. However, at the end of the 70's, the Smoothed Particle Hydrodynamics (SPH) numerical method was invented to simulate complex astrophysical problems, for which a meshless formalism is helpful, as pointed out by Gingold and Monaghan (1982). At the beginning of the 80's, this method was successfully applied to other fields of computational Physics (Monaghan 1992), in particular for rapid dynamic phenomena in fluid dynamics. Today, SPH is becoming one of the most 
popular meshless methods for fluid dynamics and is commonly applied to real free-surface flows (see e.g. Shao \& Gotoh, 2004). In particular, turbulence closures have been developed and tested by several authors. Violeau (2004) and Violeau \& Issa (2005) presented standard models and validated applications, particularly SPH forms of the traditional one and two-equation closures.

SPH is a fully Lagrangian method, which means that no computational mesh or grid is required. Thus it is particularly well adapted to the prediction of multiphase flows including floating objects. In the following, we investigate the possibility of studying the behaviour of an oil spill in the vicinity of a boom with SPH. We briefly describe the mechanism responsible for boom failure with oil leakage, supported by experiments. The SPH turbulent model is then presented and the numerical results are discussed in the case of a steady current in an open-channel and under regular waves in a wave flume.

\section{MECHANISMS OF BOOM LEAKAGE}

Floating booms, which can effectively be very efficient in some cases, can also perform a wrong way in other specific situations. In addition to diverse constraints related to the intervention itself, as well as to the meteorological conditions (security, possibilities of deployment, damage on the boom), it is possible to define five main mechanisms of failures due to the efficiency of intact booms themselves (see figure 2), when confronted to current or waves. Not considering the quality and type of the booms themselves, the occurrence of these failures is naturally determined by both the environmental conditions, and the nature and type of the oil slick.

- Entrainment: strong currents often lead to the building of an upstream wave on the contained oil slick. Associated turbulence tends then to break away oil droplets from the slicks, which are entrained under the boom. This kind of failure constitutes a strong constraint to oil booms, since it generally starts at rather low current speeds (usually estimated around $0.4 \mathrm{~m} / \mathrm{s}$ in field conditions).

- Drainage: in the case of relatively dense oils, the slick can thicken enough to overflow under the 
boom, which phenomenon is increased by the movement of water. The critical current speed for the occurrence of drainage is dependant of the depth of the skirt and the properties of the oil, but is generally higher than the critical speed for entrainment.

- Splash-over: in choppy sea conditions, some oil is likely to be splashed over the freeboard of the boom. This phenomenon is favoured by high amplitude and low length-to-height ratio waves.

- Boom submergence: when anchored in strong currents or towed at too important speeds, floating booms are likely to be submerged, in which case they suffer from a great loss of efficiency. Inflatable booms, featuring rather important buoyancy, are generally less subject to this phenomenon. However, in such cases, the real risk relies mainly in the possibility of damaging the boom, since containment failures have already generally largely started by entrainment at that stage.

- Boom planning: opposite wind and current can also cause a planning phenomenon, in which the boom flattens on the sea surface. Once again, this situation often occurs at rather higher current speeds than for entrainment.

From a fluid mechanics point of view, the first three phenomena appear the most interesting to study, since they are not relevant to the conception or floating behaviour of the boom; they will be numerically reproduced in the next sections. In this section, we try to briefly investigate the mechanisms of boom leakage by entrainment due to turbulent eddies in the case of a steady current. In the following we work in a vertical section with the notations indicated on figure 3: a steady turbulent flow characterised by a water depth $H$ and surface mean velocity $U_{s}$ with a boom of diameter $D$ and skirt depth $d$. Water (resp. oil) density and molecular kinetic viscosity are denoted by $\rho$ and $v$ (resp. $\rho_{h}$ and $v_{h}$ ). The angle $\theta$ between the skirt and the vertical axis is not considered as a parameter, since it should depend on the flow characteristics. However, one may object that $\theta$ should also be dependent on the boom mechanical characteristics; thus one should keep in mind that this assumption is one of the most important in the coming developments. With the assumptions mentioned above, dimensional 
analysis gives the critical surface velocity for which leakage occurs in the general form

$$
U_{s, c}=f\left(g, H, D, d, \rho, v, \rho_{h}, v_{h}\right)
$$

In which $g$ is the gravitational acceleration. Obviously, the boom diameter has a very small influence, as well as the water depth provided it is high enough. Vaschy-Buckingham theorem then yields

$$
\frac{U_{s, c}}{\sqrt{g d}}=f\left(\frac{U_{s, c} d}{v}, \frac{U_{s, c} d}{v_{h}}, \frac{\rho_{h}}{\rho}\right)
$$

The Reynolds number based on water viscosity is always very high, so that it has no influence (fully turbulent flow). In the case of light oil, the same conclusion occurs for the Reynolds number based on oil viscosity, so that we finally get

$$
U_{s, c}=\sqrt{g d} f\left(\frac{\rho_{h}}{\rho}\right)
$$

which means that, for a given type of oil, the critical leakage Froude number should be an unknown constant. The following mechanism can be invoked to explain equation (3) and find a good approximation of $U_{s, c}$. Consider a piece of oil of volume $V$ located on the vicinity of the boom near the water-oil interface. There are two main forces acting on it: gravity (including buoyancy) and shear stress. The latter enhances the production of turbulent eddies in the vicinity of the interface, moving the oil particle along roughly circular paths below the interface, before it comes back into the oil spill (see figure 4). This phenomenon is modelled by a production term $P$ in the equation governing the turbulent kinetic energy $k$ (see e.g. Pope, 2000, p. 125):

$$
\frac{\partial k}{\partial t}+\overline{\mathbf{u}} \cdot \nabla k=P+\nabla \cdot \mathbf{Q}_{k}-\varepsilon
$$

where $\overline{\mathbf{u}}$ is the Reynolds-averaged velocity, $\mathbf{Q}_{k}$ the turbulent kinetic energy flux and $\varepsilon$ the dissipation rate. During its stage into the water, buoyancy forces acting on the oil particle are proportional to ( $\rho-$ 
$\left.\rho_{h}\right) V$; if the power $P_{g}$ of these forces (per unit mass) is larger that kinetic energy production, then the particle will come back into the oil spill and the flow will be stable in average. Thus the instability condition reads

$$
\frac{P}{P_{g}} \geq 1
$$

It should be noticed that the term appearing in equation (5) is a kind of bulk Richardson number, giving the order of magnitude of the ratio of turbulent production to buoyancy for an oil particle moving into water. Note that if the flow was continuously stratified, the term $P_{g}$ would appear explicitly as a buoyancy term in (4). The production rate is defined by $P=-t r(\mathbf{R S})$, in which $\mathbf{R}$ is the Reynolds stress tensor and $\mathbf{S}$ the mean rate-of-strain tensor, respectively defined by

$$
\mathbf{R}=\overline{\mathbf{u}^{\prime} \otimes \mathbf{u}^{\prime}}
$$

and

$$
\mathbf{S}=\frac{1}{2}\left(\nabla \overline{\mathbf{u}}+{ }^{T} \nabla \overline{\mathbf{u}}\right)
$$

where the velocity turbulent fluctuation vector is denoted by $\mathbf{u}^{\prime}$. Here and in the following, overbars refer to turbulent Reynolds-averaged quantities. Traditional Boussinesq's model for $\mathbf{R}$ reads

$$
\mathbf{R}=\frac{2}{3} k \mathbf{I}-2 v_{T} \mathbf{S}
$$

in which $v_{T}$ is the eddy viscosity. Combining these formulae leads to the well known production form:

$$
P=v_{T} S^{2}
$$

$S$ being the scalar mean rate-of-strain defined by

$$
S=\sqrt{2 \mathbf{S}: \mathbf{S}}
$$

However, it was shown by many authors that such a quadratic dependency of production on $S$ can 
strongly overestimate $P$ in the vicinity of a rigid steady body (as for instance in the case of an impinging jet on a plane); this is mainly an effect of turbulence anisotropy, which is always bounded, while (8) gives an anisotropy tensor proportional to the rate-of-strain. In our case, this problem may occur in the considered area, where the rate-of-strain is very large near the skirt and the interface. In order to avoid this problem, Guimet and Laurence (2002) proposed the alternative general following form:

$$
P=\min \left(\sqrt{C_{\mu}}, C_{\mu} S \frac{k}{\varepsilon}\right) k S
$$

in which $C_{\mu}=0.09$. We now estimate the power of gravity received by the oil particle while it moves along a large turbulent eddy of size $L_{t}$ (turbulent integral scale). Figure 4 shows that when the oil particle moves along a semi-circle of diameter $L_{t}$ with an eddy velocity $u^{\prime}$, the power of gravity is given by

$$
P_{g}=\int_{-\pi / 2}^{\pi / 2}\left(\rho_{h}-\rho\right) V \mathbf{g} \cdot u^{\prime} \mathbf{e}_{\theta} d \theta=2\left(\rho-\rho_{h}\right) V g u^{\prime}
$$

Therefore, the ratio of $P$ to the power of gravity forces (per unit mass) reads

$$
\frac{P}{P_{g}}=\frac{\min \left(\sqrt{C_{\mu}}, C_{\mu} S \frac{k}{\varepsilon}\right) k S}{2\left(\rho-\rho_{h}\right) g u^{\prime} / \rho_{h}}
$$

An oil particle will be pulled out off the oil layer and will pass under the skirt if equation (5) is satisfied, thus if (13) exceed unity. Assuming that turbulence is roughly isotropic, an order of magnitude of velocity fluctuations characterising large scales can be chosen as $u^{\prime}=(2 k / 3)^{1 / 2}$. Thusn entrainment of oil particles will occur if

$$
\min \left(\sqrt{C_{\mu}}, C_{\mu} S \frac{k}{\varepsilon}\right) \frac{S \sqrt{k}}{\alpha g} \geq \sqrt{\frac{8}{3}} \approx 1.63
$$


where the relative density excess $\alpha$ is defined as

$$
\alpha=\frac{\rho}{\rho_{h}}-1
$$

Let us now try to simplify equation (14) by making additional assumptions. Firstly, using the quadratic form of production (9) in place of (11) would give a more simple criterion. Indeed, assuming that turbulence is in a local equilibrium gives $P \cong \varepsilon$ from equation (4). In this particular case, the two forms of production in equation (11) coincide, and $P$ can be re-written as

$$
P=\sqrt{C_{\mu}} S k
$$

Then, equation (14) takes the form

$$
\sigma \equiv \frac{S \sqrt{k}}{\alpha g} \geq \sqrt{\frac{8}{3 C_{\mu}}} \approx 5.44
$$

which defines the control parameter $\sigma$. Eq. (17) can be considered as a criterion for oil entrainment; more precisely entrainment should occur when $\sigma$ goes beyond the critical value 5.44 somewhere in the vicinity of the oil-water interface. In a second step, an estimation of $k$ can be achieved by assuming that the dissipation rate $\varepsilon$ in equation (4) is related to $k$ by a dimensional relation involving a mixing length $L_{m}$ :

$$
\varepsilon=C_{\mu}^{3 / 4} \frac{k^{3 / 2}}{L_{m}}
$$

where $L_{m}$ is close to the eddy size $L_{t}$. Then, assuming $P \cong \varepsilon$ and combining the latter relation with (16), one gets

$$
k=\frac{\left(L_{m} S\right)^{2}}{\sqrt{C_{\mu}}}
$$

The condition (17) then reads 


$$
\frac{L_{m} S^{2}}{\alpha g} \geq \frac{1}{C_{\mu}^{1 / 4}} \sqrt{\frac{8}{3}} \approx 2.98
$$

Eventually, one can estimate an order of magnitude of $S$ as $2 U_{s} / \delta$, where $\delta$ is the approximate thickness of the oil layer, while $L_{m}$ is close to $\delta$ (see figure 4). Given these last and very rough approximations, equation (20) finally reads

$$
\frac{U_{s}}{\sqrt{\alpha g d}} \geq \frac{1}{\left(6 \sqrt{C_{\mu}}\right)^{1 / 4}} \sqrt{\frac{\delta}{d}} \approx 0.86 \sqrt{\frac{\delta}{d}}
$$

revealing that the critical Froude number is proportional to the square root of $\alpha$. The critical velocity thus appears in a form according to equation (3), with $f\left(\rho_{h} / \rho\right)=\alpha^{1 / 2}$. The experiments carried out by the CEDRE reveal a ratio $\delta / d$ ranging around 0.25 (see e.g. figure 6). Thus, with a skirt depth $d=$ $0.75 \mathrm{~m}$ and $\alpha=0.18$ (typical values, as presented later), equation (21) gives the order of magnitude $U_{s, c}=0.5 \mathrm{~m} / \mathrm{s}$. Although the last equation was reached after many approximations, we will see in the next section that it provides a satisfactory value. However, the general condition (17) will be considered as more realistic; its effectiveness will be tested in the following against our SPH numerical simulations.

\section{EXPERIMENTAL SETUP}

In order to observe the above-mentioned processes, we considered a series of experiments carried out at the University of La Rochelle, in the LEPATB facilities. This $20 \mathrm{~m}$ long canal is equipped with a motorised chariot which allows the progressive evolution of the sea-current velocity. 1/15 scale models of floating anti-hydrocarbon booms were created; they are used to measure the pressures which the booms undergo and the risk of oil leakage (see figure 5). For each experimental study we considered a Froude similarity, which gives a range of experimental velocities of a few centimetres 
per second. The model booms have a diameter of $37 \mathrm{~mm}$ and a skirt height of $51 \mathrm{~mm}$. With the help of hydrodynamic gauges, three extensometric relays, and an data acquisition system, we measured the drag, lift and angular momentum coefficients of the boom as a function of the current velocity $U_{s}$ and the skirt inclination $\theta$. An experimental setup was specially created to confine oil, to visualise different regime flows, and to determine leak types and leakage velocity thresholds. We used colza oil to simulate heavy oil and Kerdan to simulate light oil. We also coloured these substitute fluids with red organol in order to create a better contrast against water. These confinement and leak visualisations are possible thanks to a camera and an under water mirror placed close to the boom. Leaks of contaminants can be observed, first as small drops, then as larger quantities and finally by overflow. The oil is recovered and the water is cleared after each experiment (more details on this experimental setup is available in Abed-Meraïm, 2006).

Our experiments allowed us to observe and understand the mechanisms of oil leakage by entrainment (figure 6) and drainage (figure 7). In the case of entrainment, one can clearly observe that oil droplets are attracted under the oil spill by turbulent fluctuations, as mentioned earlier. The control parameter $\sigma$ was not calculated from our measurements, but the critical velocity for oil entrainment (in nature) was estimated as $0.7 \mathrm{~m} / \mathrm{s}$, which is above the estimated critical value $U_{s, c}=0.5 \mathrm{~m} / \mathrm{s}$ given in section 2 . This may be due to the rigidity of the experimental skirt, arbitrarily set in a vertical position (as it can be seen on figures 6 and 7). However, this estimation confirms the order of magnitude of $U_{s, c}$ given in section 2. In the following, the presented experiments will be used to make a qualitative validation of the numerical simulations.

\section{SPH EQUATIONS FOR TURBULENT FLOWS}

In this section, we briefly present the equations of the SPH numerical method for fluid dynamics; for more details the reader can refer to Monaghan (1992) and Violeau \& Issa (2006). The SPH formalism 
is based on the idea that a flow can be considered as a set of bulk parts of moving fluid referred to as "particles". Each particle $a$, located at $\mathbf{r}_{a}$, has a constant mass $m_{a}$ and carries a density $\rho_{a}$, Reynoldsaveraged pressure $\bar{p}_{a}$ and velocity vector $\overline{\mathbf{u}}_{a}$, dynamic (resp. kinematic) viscosity $\mu_{a}$ (resp. $v_{a}=\mu_{a} /$ $\rho_{a}$ ), and more generally different physical quantities (e.g. temperature or energy). All these quantities evolve according to governing equations, which are written in terms of fluxes between particles.

At the heart of SPH, any function $A$ relative to a point $\mathbf{r}$ is written as a convolution product with an interpolant kernel function $w_{h}$ :

$$
A(\mathbf{r})=\int_{\Omega} A\left(\mathbf{r}^{\prime}\right) w_{h}\left(\mathbf{r}-\mathbf{r}^{\prime}\right) d r^{\prime}+O(h)
$$

The summation is extended to the entire domain $\Omega$ and the parameter $h$, namely the smoothing length, will be discussed later. Note that equation (22) would be mathematically exact if the Dirac distribution was considered in place of $w_{h}$. The transition to a discrete domain is achieved by approximating (19) with a Riemann summation or by using the Monte-Carlo formula, considering the case of a spherical kernel (i.e. $w_{h}$ only depends on the distance between particle pair):

$$
A\left(\mathbf{r}_{a}\right)=\sum_{b} \frac{m_{b}}{\rho_{b}} A_{b} w_{h}\left(r_{a b}\right)
$$

where $b$ is a dummy subscript referring to each particle present in the domain, the infinitesimal volume $d r^{\prime}$ being replaced by the volume $m_{b} / \rho_{b}$ of particle $b . A_{b}$ is the value of $A$ at the location $\mathbf{r}_{b}$ of particle $b$ and $r_{a b}$ denotes the distance between $a$ and $b$. Equation (23) is called the SPH interpolation. It appears that it is differentiable, provided the kernel is differentiable too; if the function $A$ corresponds to a scalar field, the basic form of its gradient relative to particle $a$ can then be obtained by taking the gradient of (23):

$$
\nabla A\left(\mathbf{r}_{a}\right)=\sum_{b} \frac{m_{b}}{\rho_{b}} A_{b} \nabla_{a} w_{a b}
$$


in which the symbolic expression $\nabla_{a}$ denotes the gradient of the kernel taken with respect to the coordinates of particle $a$, while $w_{h}\left(r_{a b}\right)$ is written $w_{a b}$ for simplicity. This can also be done for any differential operator such as the divergence of a vector field. Therefore, the SPH formalism allows the possibility of estimating any differential operator by using the kernel, without using any computational grid. A very usual kernel example in two dimensions is the $4^{\text {th }}$ order spline, used in most of the applications of the present work:

$$
w_{h}\left(r_{a b}\right) \equiv f(q)=\frac{\beta}{h^{2}} \begin{cases}\left(\frac{5}{2}-q\right)^{4}-5\left(\frac{3}{2}-q\right)^{4}+10\left(\frac{1}{2}-q\right)^{4} & \text { if } 0 \leq q \leq 0,5 \\ \left(\frac{5}{2}-q\right)^{4}-5\left(\frac{3}{2}-q\right)^{4} & \text { if } 0,5 \leq q \leq 1,5 \\ \left(\frac{5}{2}-q\right)^{4} & \text { if } 1,5 \leq q \leq 2,5 \\ 0 & \text { otherwise }\end{cases}
$$

$q$ being defined as the ratio $r_{a b} / h$ and $\beta=96 / 1199 \pi$ in two dimensions, to ensure kernel normalization. An important feature of (25) is that $w_{h}$ is designed with a compact support proportional to $h$, as showed on figure 8 . The smoothing length $h$ is commonly proportional to the initial particle spacing, so that each particle interacts with a finite number of neighbour particles. In SPH equations like (24), it means that the summation runs over a reduced number of particles $b$, keeping the algorithm proportional to the particle number. A link table between particles is thus established at each time step to keep the algorithm efficient in terms of computational time.

Given these basic tools, the Reynolds-averaged Navier-Stokes equations can be written in SPH formalism. The continuous Lagrangian forms of these equations for a weakly compressible flow are

$$
\begin{gathered}
\frac{d \overline{\mathbf{u}}}{d t}=-\frac{1}{\rho} \nabla \bar{p}+v \Delta \overline{\mathbf{u}}-\frac{1}{\rho} \nabla \cdot(\rho \mathbf{R})+\mathbf{g} \\
\frac{d \rho}{d t}=-\rho \nabla \cdot \overline{\mathbf{u}}
\end{gathered}
$$


where $\mathbf{g}$ is the acceleration of gravity (low density variations are assumed to have no effect in the momentum equation (26)). Note that eq. (26) and (27) are valid in both water and oil, although the notations regarding density and molecular viscosity here refer to water. Assuming the validity of Boussinesq's model (8), given the general SPH gradient approximation (24) and applying similar considerations to the divergence operator, possible SPH forms of (26) and (27) are

$$
\begin{gathered}
\frac{d \overline{\mathbf{u}}_{a}}{d t}=-\sum_{b} m_{b}\left(\frac{\bar{p}_{a}}{\rho_{a}^{2}}+\frac{\bar{p}_{b}}{\rho_{b}^{2}}-\frac{\mu_{e, a}+\mu_{e, b}}{\rho_{a} \rho_{b}} \frac{\overline{\mathbf{u}}_{a b}}{r_{a b}^{2}} \mathbf{r}_{a b} \cdot\right) \nabla_{a} w_{a b}+\mathbf{g} \\
\frac{d \rho_{a}}{d t}=\sum_{b} m_{b} \overline{\mathbf{u}}_{a b} \cdot \nabla_{a} w_{a b}
\end{gathered}
$$

where $\mathbf{r}_{a b}=\mathbf{r}_{a}-\mathbf{r}_{b}, \overline{\mathbf{u}}_{a b}=\overline{\mathbf{u}}_{a}-\overline{\mathbf{u}}_{b}$ and $\mu_{e, a}=\mu_{a}+\mu_{T, a}$. Therefore, each particle $a$ is affected by a dynamic eddy viscosity, referred to as $\mu_{T, a}=\rho_{a} v_{T, a}$. Since we deal here with nearly incompressible flows, we assume that $\bar{\rho}_{a} \approx \rho_{a}$. Equation (28) is a momentum equation, used to compute particle velocity, while (29) is an SPH form of the continuity equation, giving particle density. Both are integrated in time through an explicit Euler scheme, and particle positions are obtained by velocity integration. Pressure can be estimated from a compressible equation of state:

$$
\bar{p}_{a}=\frac{\rho_{0} c_{0}^{2}}{\gamma}\left[\left(\frac{\rho_{a}}{\rho_{0}}\right)^{\gamma}-1\right]
$$

in which $\rho_{0}$ is a reference density, $c_{0}$ a numerical speed of sound and $\gamma=7$ for water (Monaghan, 1994). If $c_{0}$ is high enough, equation (30) is suitable for a weakly compressible flow. However, for numerical reasons, the value of $c_{0}$ in the model is not the true speed of sound; indeed, the time step is usually subject to some stability constraints:

$$
\delta t=\min \left(0.4 \frac{h}{c_{0}}, 0.25 \min _{a} \sqrt{\frac{h}{\gamma_{a}}}, 0.125 \min _{a} \frac{\rho_{a} h^{2}}{\mu_{a}}\right)
$$


where $\gamma_{a}$ is the modulus of the acceleration of particle $a$. The speed of sound appearing in the first constraint would yield very small time steps with the physical value of $c_{0}$. Therefore, it is chosen as ten times the maximum flow speed, keeping the relative density fluctuations less than $1 \%$. With this approximation, SPH appears to be a weakly compressible method.

Particle eddy viscosity are estimated here through the traditional one-equation $k-L_{m}$ model developed in the context of Eulerian numerical methods, and adapted to the SPH context (Violeau, 2004; Violeau $\&$ Issa, 2006). Firstly, one defines for each particle $a$ a turbulent kinetic energy $k_{a}$ and a mixing length $L_{m, a}$ (here considered as equal to the particle size) and assumes that equation (18) is valid for all the particles. Then, an additional equation is required to calculate $k_{a}$ at each time step. An SPH form of (4) is written as:

$$
\frac{d k_{a}}{d t}=P_{a}-\varepsilon_{a}+\sum_{b} m_{b} \frac{\mu_{k, a}+\mu_{k, b}}{\rho_{a} \rho_{b}} \frac{k_{a b}}{r_{a b}^{2}} \mathbf{r}_{a b} \cdot \nabla_{a} w_{a b}
$$

where $\mu_{k, a}=\mu_{a}+\mu_{T, a} / \sigma_{k}$ is a dynamic diffusivity for $k$ and $\sigma_{k}$ a constant Schmidt number. Equation (32) involves particle production $P_{a}$ defined by equation (11) and turbulent diffusion (in which $k_{a b}=k_{a}$ $\left.-k_{b}\right)$. Note that a more sophisticated model (e.g. $\left.k-\varepsilon\right)$ could be used to avoid the use of this parameter; however, the large computational time required for the presented applications make this simpler model more attractive. The scalar rate-of-strain $S_{a}$ relative to particle $a$ is estimated by the following SPH approximation (see Violeau \& Issa, 2006):

$$
S_{a}^{2}=-\frac{1}{2} \sum_{b} m_{b} \frac{\rho_{a}+\rho_{b}}{\rho_{a} \rho_{b}} \frac{\bar{u}_{a b}^{2}}{r_{a b}^{2}} \mathbf{r}_{a b} \cdot \nabla_{a} w_{a b}
$$

We use here the set of constant values recommended by Launder and Spalding (1972): $C_{\mu}=0.09$ and $\sigma_{k}=1.0$

Solid boundaries are modelled here with fixed mirror particles. The main idea is to model walls with 
"wall particles" and several layers of "ghost particles" (see figure 8, right picture), density of which is prescribed by symmetry when computing particle density through equation (29). Thus, equation (30) keeps pressure forces symmetric with respect to the wall, ensuring an artificial Neumann condition regarding pressure. Dirichlet wall boundary conditions for kinetic energy and mean velocity are specified as

$$
\begin{aligned}
& k_{a}=\frac{u_{*, a}^{2}}{\sqrt{C_{\mu}}} \\
& \frac{\bar{u}_{a}}{u_{*, a}}=\frac{1}{\kappa} \ln \frac{\delta_{*}}{k_{s}}+C_{*}
\end{aligned}
$$

for all particles located on solid boundaries. In the traditional log-law appearing in (34), $\kappa=0.41$ is von Karman's constant and $C_{*}=8.5$, while $k_{\mathrm{s}}$ is the equivalent bed roughness; $\delta *$ is an arbitrary distance chosen small enough in comparison to the particle size, but larger than the roughness. In the following, the value of $\delta *$ was chosen as a few millimeters, but have no visible effects on the results. The value of shear velocity and $u_{*, a}$ attached to a wall particle $a$ is estimated from the wall velocity gradient.

Specific free surface boundary conditions are not considered here, provided the lack of particles in the vicinity of a free surface makes all quantities rapidly decreasing, which is enough for numerical stability and physical meaning. In particular, pressure, eddy viscosity and turbulent kinetic energy vanish near the free surface.

\section{APPLICATION TO A BOOM}

The abovementioned SPH model was developed at LNHE in a 2D vertical code, and validated on several test cases (Violeau \& Issa, 2006). For the purpose of the present study, the simulation of a rigid body motion is achieved by the following way (see Monaghan et al., 2003 for more details). The 
boom is made of particles receiving forces from fluid particles. One computes the total force $\mathbf{F}$ received by the boom (including pressure forces, viscous forces and gravity), and make it move according to Newton's third law as

$$
M \frac{d \mathbf{U}}{d t}=\mathbf{F}
$$

where $M$ and $\mathbf{U}$ indicate the boom mass and velocity. According to equation (28), the force is simply

$$
\mathbf{F}=-\sum_{a, b} m_{a} m_{b}\left(\frac{\bar{p}_{a}}{\rho_{a}^{2}}+\frac{\bar{p}_{b}}{\rho_{b}^{2}}-\frac{\mu_{e, a}+\mu_{e, b}}{\rho_{a} \rho_{b}} \frac{\overline{\mathbf{u}} a b}{r_{a b}^{2}} \mathbf{r}_{a b} \cdot\right) \nabla_{a} w_{a b}+M \mathbf{g}
$$

where the summation runs over all fluid particles $b$ and boom particles $a$. In the following applications, however, it should be noted that the particles corresponding to the boom were set with the same reference density as those of water, to avoid numerical instabilities. Thus, the true boom density (here chosen as $13 \mathrm{~kg}$ per meter) is only taken into account through the boom mass $M$ in equation (35). The boom will be represented in a 2D-vertical section like in figure 9. Thus, its motion is restricted to the vertical axis, provided that there is an equilibrium between the horizontal drag force due to the fluid and the force exerted by anchorages. For the same reason, angular motion will not be considered, the angle $\theta$ between the skirt and the vertical axis depending on the choice of the vertical section (see figure 10). Three values of the angle were examined: $-10^{\circ},+10^{\circ}$ and $+40^{\circ}$.

Modelling an oil spill with SPH is rather easy: it consists of defining two kinds of particles, each having its own reference density and molecular viscosity (e.g. $v_{a}=v$ or $\left.v_{h}\right)$. SPH has proved its ability to reproduce multiphase flows (Colagrossi \& Landrini, 2003) and may not present any additional problems herein. Three kinds of oil were tested: a heavy one, a lighter one and an emulsion, the physical characteristics of which are indicated in table 1 (the density of water is set to $1000 \mathrm{~kg} / \mathrm{m}^{3}$ ). In case of the heavy - and thus very viscous - oil, turbulence can be switched off for all oil particles simply by setting the eddy viscosity to zero. This is done also in the case of the emulsion, which 
consists of a mixture, for which the molecular viscosity is not a constant but depends on the scalar rate-of-strain $S$. Laboratory experiments were carried out at CEDRE in Brest to provide the following constitutive law:

$$
\frac{v_{h}}{v_{r e f}}=\exp \left[-\left(\frac{S}{S_{0}}\right)^{\eta}\right]
$$

with $v_{\text {ref }}=0.272 \mathrm{~m}^{2} \mathrm{~s}^{-1}, S_{0}=6.85 \mathrm{~s}^{-1}$ and $\eta=0.5033$ (see figure 11). The values of viscosities indicate that The values of viscosities indicate that this oil flow will stay laminar, while turbulence will certainly develop in the first two cases.

Two kinds of flows were tested, both two-dimensional in a vertical plane: a steady current in an open channel and wave motion in a wave flume. In the first case, the channel is periodic, meaning that it must be long enough to avoid artificial periodicity effect: the length is $\ell=16 \mathrm{~m}$ and the water depth at rest $H=2.5 \mathrm{~m}$ (see figure 12). The flow is driven by a horizontal force $\mathbf{F}_{D}$ prescribed to each fluid particle in the r-h-s of equation (28). This force is updated at each time step in order to prescribe a constant flow rate; the ability of SPH to model such a turbulent steady flow was shown by Violeau \& Issa (2006). Two values of upstream surface velocities $U_{s}$ were examined. Assuming that the traditional log-law is valid at a certain distance upstream to the boom, the relation between $U_{s}$ and the bed shear velocity $u_{*}$ is

$$
\frac{U_{s}}{u_{*}}=\frac{1}{\kappa} \ln \frac{H}{k_{s}}+C_{*}
$$

Where the bottom roughness $k_{s}$ was chosen as $1 \mathrm{~mm}$. Let us consider the whole fluid domain (in two dimensions) of surface $\Sigma$ (per unit length in the direction perpendicular to figure 12). A momentum balance then gives $\mathbf{F}_{D}$ : 


$$
\rho \mathbf{F}_{D} \Sigma=-\oint_{\partial \Omega_{F} \cup \partial \Omega_{B}}^{-} \cdot \mathbf{n} d \Gamma
$$

where $\partial \Omega_{F}$ and $\partial \Omega_{B}$ are the fluid and boom boundary, respectively, and $\bar{\tau}$ the total stress tensor.

Thus the magnitude of the driving force is

$$
\rho F_{D} \Sigma=\rho u_{*}^{2} \ell+F_{B}
$$

where $F_{B}$ is the drag force (per unit length) experienced by the boom, mainly on its skirt ( $u *$ was assumed to be spatially constant). Finally, one can introduce the boom drag coefficient $C_{D}$ to write

$$
F_{D} \approx\left[\left(\frac{1}{\kappa} \ln \frac{H}{k_{s}}+C_{*}\right)^{-2}+\frac{d}{2 \ell} C_{D}\right] \frac{U_{s}^{2}}{H}
$$

where we used the approximation $\Sigma=H \ell$. Equation (41) gives $F_{D}$ as a function of the required surface velocity $U_{s}$. Table 2 indicates the set of parameter tested in this first case.

The drag coefficient (assumed to be constant for high Reynolds number flows) was estimated from experiments carried out in the LEPTAB facilities presented above. Table 3 gives the values of drag coefficients, shear velocities and numerical driving force for each configuration, calculated from equation (41).

The second case, namely the wave flume, consists of a $25 \mathrm{~m}$ long canal with a flat bed and a gentle slope (see figure 13), ended by a dyke to avoid too much wave reflection. At the opposite side, an oscillating paddle generates monochromatic waves of given amplitude according to table 4 . The still water height is $2 \mathrm{~m}$, while the wave period was fixed to $T_{w}=4 \mathrm{~s}$. The emulsion was not considered in this case. The ability of SPH to predict wave motion was pointed out by several authors (see e.g. Shao \& Gotoh, 2004).

\section{RESULTS IN THE OPEN CHANNEL}


The SPH simulations show that the model is able to reproduce at least two types of oil leakage: entrainment (figure 14) and drainage (figure 15). On a qualitative point of view, the entrainment process is correctly reproduced when compared to our experiments, as pointed out by figure 16. Figure 17 shows the computed spatial distribution of the maximum (in time) value of the $\sigma$ parameter as defined by equation (17) for a light oil and skirt angle $\theta=+10^{\circ}$, for two values of the surface velocity $\left(U_{s}=0.3 \mathrm{~m} / \mathrm{s}\right.$ and $\left.0.7 \mathrm{~m} / \mathrm{s}\right)$. Obviously, the higher velocity leads to higher values of the scalar mean rate-of-strain $S$, thus higher values of production $P$ (see eq. (16)) and turbulent kinetic energy $k$, and then yields higher values of $\sigma$ as well. Numerically, one observes that entrainment occurs on the second case $\left(U_{s}=0.7 \mathrm{~m} / \mathrm{s}\right)$ but not on the first $\left(U_{s}=0.3 \mathrm{~m} / \mathrm{s}\right)$. Figure 18 shows the computed maximum (in space) of $\sigma$ as a function of time. Interestingly, in the case $U_{s}=0.3 \mathrm{~m} / \mathrm{s}$, this parameter is almost always bounded by the critical threshold 5.44 predicted by (eq. (17)), while in the case $U_{s}=$ $0.7 \mathrm{~m} / \mathrm{s}$ the spatial maximum of $\sigma$ goes beyond this threshold twice, once at the beginning of the computation, the second time around $t=10 \mathrm{~s}$ after the beginning. Indeed, during the first stages of the computation, velocity variations are very high under the boom skirt, leading to high values of $\sigma$ in this area (see figure 17), until the flow gets steady. Conversely, at $t=10 \mathrm{~s}$, the maximum is located near the oil-water interface, and entrainment is begins in our simulations. This confirms that the theoretical developments presented in section 2 are pertinent and provide a good criterion for oil entrainment.

Extensive simulations have been carried out in order to study the effect of various parameters on the behaviour of oil and risk of oil leakage. Figure 19 shows the effect of the type of oil; one can see that the heavy oil and the emulsion both lead to drainage. Figure 20 shows the influence of the skirt angle $\theta$; entrainment remains for $\theta=+10^{\circ}$ while drainage occurs when $\theta=+40^{\circ}$. Table 5 summarizes the computed results.

We saw earlier that an order of magnitude of the critical velocity for entrainment is given by $U_{s, c}=0.5$ $\mathrm{m} / \mathrm{s}$ for $\alpha=0.18$ (light oil). Eq. (21) yields to the value $U_{s, c}=0.09 \mathrm{~m} / \mathrm{s}$ for heavy oil $(\alpha=0.005)$. 
These estimations are also roughly confirmed by our numerical tests; indeed table 5 shows that oil leakage always appears in the case of a heavy oil (our tested velocities are always above $0.09 \mathrm{~m} / \mathrm{s}$, as shown of table 2), while leakage appears only for $U_{s}=0.7 \mathrm{~m} / \mathrm{s}$ in the case of light oil. However, one may notice that the criterion given by eq. (21) has been based on considerations regarding entrainment, while drainage occurs in most circumstances when $U_{s}=0.7 \mathrm{~m} / \mathrm{s}$. A better estimation of $U_{s, c}$ would thus require a more exhaustive set of simulations or experiments. One should also notice that our criterion fails in the case of light oil when the skirt angle is $\theta=-10^{\circ}$, for which there is no leakage at all (even when $U_{s}=0.7 \mathrm{~m} / \mathrm{s}$ ). This is probably be due to the fact that we neglected the effect of skirt angle in our theoretical estimations.

In the case of emulsion, eq. (21) also gives a critical speed $U_{s, c}=0.09 \mathrm{~m} / \mathrm{s}$. However, there is no leakage when $U_{s}=0.3 \mathrm{~m} / \mathrm{s}$; this tends to indicate that the high molecular viscosity of the emulsion limits the possibility of oil entrainment by turbulent eddies.

\section{RESULTS IN THE WAVE FLUME}

In nature, the orbital velocities can significantly increase the rate-of-strain and thus allow oil leakage even under a small current. However, modelling combined waves and current with our model leads to some difficulties; it was then decided to focus separately on waves. No experiments were carried out in this case. Based on the fact that the tests in the steady open-channel gave satisfactory results, the wave flume model was used to investigate the behaviour of the boom and the oil spill under wave motion alone.

Figure 21 shows that a rather large wave amplitude $\left(H_{w}=1.0 \mathrm{~m}\right)$ can lead to leakage by splash-over with the light oil and a skirt angle $\theta=-10^{\circ}$. Figure 22 presents a similar phenomenon in the case of heavy oil. Strangely, in the case of the light oil, the skirt angle $\theta=+10^{\circ}$ leads to a completely different motion of the oil spill, which stays upstream of the boom (figure 23). This results from the fact that 
the lift coefficient of the boom is in this case different; the boom tends to follow the wave motion and keeps the oil near its original location; no leakage is observed in this case. The effect of skirt angle on the behaviour of the heavy oil spill is slightly different: the negative angle prevents from splash-over but leads to a leakage by drainage (figure 24).

Generally speaking, no leakage occurs for a wave height of $0.5 \mathrm{~m}$, while it always occurs when $H_{w}=$ $1.0 \mathrm{~m}$ (see figure 25 and table 5). This tends to indicate that in the absence of steady current, a critical wave height $H_{w, c}$ may exist between $0.5 \mathrm{~m}$ and $1.0 \mathrm{~m}$; however, one should remember that the influence of wave period was not tested (see table 4). Finally, figure 26 shows the influence of the oil density. The light oil sometimes keeps stable even with the higher wave height. Despite these interesting considerations, a larger range of parameters would be necessary to clearly identify the mechanisms of oil leakage under waves and the values of critical wave height and period.

\section{CONCLUSIONS}

A first attempt of modelling a floating boom and an oil spill was carried out through the Smoothed Particle Hydrodynamics (SPH) numerical method. Two kinds of turbulent flows were considered: a steady open-channel flow and regular waves in a flume. For both models the behaviour of the oil and the risk of leakage was observed (results are summarized in table 5).

In the case of the open-channel steady flow, it appears that the critical value of surface velocity for which leakage occurs stands between 0.3 and $0.7 \mathrm{~m} / \mathrm{s}$, which is confirmed by a rough theoretical estimation. Moreover, the mechanisms of leakage by entrainment can be explained by a theoretical analysis based on turbulence-buoyancy interactions. This leads to a more accurate criterion for entrainment which is perfectly confirmed by the numerical experiments. However, a more exhaustive series of tests would be necessary to give a more accurate estimation of the critical velocity. Numerically, the effect of turbulence closure should also be investigated (e.g. a $k-\varepsilon$ model, see 
Violeau \& Issa 2006). Further tests will highlight this point.

In the case of wave flume, no validation from experiments could be made. However, it appears that leakage occurs from a critical wave height standing between 0.5 and $1.0 \mathrm{~m}$, for the tested wave period of $4 \mathrm{~s}$. Here again, a more extended panel of numerical tests would allow a better knowledge of the mechanisms. The case of random waves should also be investigated in the future.

Generally speaking, one may point out some drawbacks of the proposed method. Surface tension forces, which can be of some importance for the mechanism of oil entrainment through droplets, was neglected here, although some authors have shown that they can be considered with no difficulty in SPH (see e.g. Monaghan, 1995). One could also mention the fact that our model is two-dimensional, whereas experiments show a three-dimensional behaviour deeply connected with the structure of turbulence and oil surface tension coefficient. However, despite the ability of SPH to run in three dimensions, the huge number of particles that would be required for such a simulation keeps this possibility cumbersome. Further tests should focus on surface tension modelling and combined wave and currents.

\section{ACKNOWLEDGEMENTS}

This work has been carried out in the context of the SIMBAR French national project, under financial support of the French Ministry of Environment through the RITMER network.

\section{REFERENCES}

Abed-Meraïm, K., 2006. Etude expérimentale de l'écoulement à surface libre autour de maquettes de barrages antipollution marine. Proc. Journées de l'AUM - AFM, La Rochelle, France (in press).

Colagrossi, A., and Landrini, M., 2003. Numerical simulation of interfacial flows by Smoothed Particle Hydrodynamics. J. Comput. Phys. 191: 448-475. 
Gingold, R.A., and Monaghan, J.J., 1982. Kernel estimates as a basis for general particle methods in hydrodynamics. J. Comput. Phys. 46: 429-453.

Guimet, V, and Laurence, D., 2002. A linearised turbulent production in the $\mathrm{k}-\varepsilon$ model for engineering applications. Proc. $5^{\text {th }}$ Int. Symp. Eng. Turb. Modelling and Measurements, Mallorca, Spain, pp. 157-166.

Launder, B.E., and Spalding, D.B., 1972. Mathematical models of turbulence. Academic Press, London.

Monaghan, J.J., 1992. Smoothed Particle Hydrodynamics. Ann. Review Astroph. 30: 543-74.

Monaghan, J.J., 1994. Simulating free surface flows with SPH. J. Comput. Phys. 110: 399-406.

Monaghan, J.J., 1995. An SPH formulation of surface tension. Applied Mathematics Reports and Preprints 95/44, University of Monash, Australia.

Monaghan, J.J., Kos, A., and Issa, N., 2003. Fluid motion generated by impact. J. Waterways, Port, Coastal and Ocean Eng., 129(6): 250-259.

Pope, S.B., 2000. Turbulent flows. Cambridge University Press.

Shao, S, and Gotoh, H., 2004. Simulating coupled motion of progressive wave and floatting curtain wall by SPH-LES model. Coastal Eng. $46: 171-202$.

Violeau, D. (2004). One and two-equations turbulent closures for Smoothed Particle Hydrodynamics. Proc. $6^{\text {th }}$ Int. Conf. Hydroinformatics, Singapore, pp. 87-94.

Violeau, D., and Issa, R, 2006. Modelling complex turbulent free surface flows with the SPH gridless method: an overview. Int. J. Num. Meth. Fluids (in press).

\section{TABLES}

Table 1

List of parameters of the three types of oil considered in this study. 


\begin{tabular}{llll}
\hline Type of oil & $\rho_{h} / \rho$ & $\alpha$ & $v\left(\mathrm{~m}^{2} \mathrm{~s}^{-1}\right)$ \\
\hline Light & 0.850 & 0.176 & $3.3210^{-6}$ \\
Heavy & 0.995 & 0.0050 & $3.010^{-2}$ \\
Emulsion & 0.995 & 0.0050 & See equation (37) \\
\hline
\end{tabular}

Table 2

Set of parameters considered for the numerical simulation of the open-channel flow. In each case are listed the skirt angles $\theta$ considered in this study.

\begin{tabular}{llll}
\hline$U_{s}(\mathrm{~m} / \mathrm{s})$ & Light oil & Heavy oil & Emulsion \\
\hline 0.3 & $-10^{\circ},+10^{\circ},+40^{\circ}$ & $-10^{\circ},+10^{\circ},+40^{\circ}$ & $-10^{\circ},+10^{\circ}$ \\
0.7 & $-10^{\circ},+10^{\circ},+40^{\circ}$ & $-10^{\circ},+10^{\circ},+40^{\circ}$ & $-10^{\circ},+10^{\circ}$ \\
\hline
\end{tabular}

Table 3

Numerical simulation of the open-channel flow: values of the boom drag coefficient, the shear velocity and the driving force for each considered configuration.

\begin{tabular}{lllll}
\hline$U_{s}(\mathrm{~m} / \mathrm{s})$ & $u_{*}(\mathrm{~cm} / \mathrm{s})$ & $\theta\left(^{\circ}\right)$ & $C_{D}$ & $F_{D}\left(\mathrm{~m} . \mathrm{s}^{-2}\right)$ \\
\hline 0.3 & 1.09 & -10 & 1,50 & $1.3110^{-3}$ \\
& & +10 & 1,64 & $1.4310^{-3}$ \\
& +40 & 1,06 & $9.4210^{-4}$ \\
\hline 0.7 & -10 & 1,50 & $7.1510^{-3}$ \\
& 2.54 & +10 & 1,64 & $7.7910^{-3}$ \\
& & +40 & 1,06 & $5.1310^{-3}$ \\
\hline
\end{tabular}

Table 4

Set of parameters considered for the numerical simulation of the wave flume. In each case are listed the skirt angles $\theta$ considered in this study.

\begin{tabular}{llll}
\hline$H_{w}(\mathrm{~m})$ & Light oil & Heavy oil & Emulsion \\
\hline 0.5 & $-10^{\circ},+10^{\circ},+40^{\circ}$ & $-10^{\circ},+10^{\circ}$ & - \\
1.0 & $-10^{\circ},+10^{\circ},+40^{\circ}$ & $-10^{\circ},+10^{\circ}$ & - \\
\hline
\end{tabular}


Table 5

Type of oil leakage obtained from numerical tests with the present SPH model, for all tested configurations.

\begin{tabular}{llllll}
\hline \multicolumn{4}{c}{ Steady open channel flow } & Wave flume & \\
\hline Oil & Angle & $U_{s}=0.3 \mathrm{~m} / \mathrm{s}$ & $U_{s}=0.7 \mathrm{~m} / \mathrm{s}$ & $H_{w}=0.5 \mathrm{~m}$ & $H_{w}=1.0 \mathrm{~m}$ \\
\hline Light & $\theta=-10^{\circ}$ & no leakage & no leakage & no leakage & splah-over \\
& $\theta=+10^{\circ}$ & no leakage & entrainment & no leakage & no leakage \\
& $\theta=+40^{\circ}$ & no leakage & drainage & no leakage & drainage \\
\hline Heavy & $\theta=-10^{\circ}$ & $\begin{array}{l}\text { entrainment then } \\
\text { drainage }\end{array}$ & drainage & no leakage & splah-over \\
& & & no leakage & drainage \\
& $\theta=+10^{\circ}$ & $\begin{array}{l}\text { entrainment then } \\
\text { drainage }\end{array}$ & drainage & not tested & not tested \\
& $\theta=+40^{\circ}$ & entrainement and \\
drainage & drainage & not tested & not tested \\
Emulsion & $\theta=-10^{\circ}$ & no leakage & drainage & not tested & not tested \\
& $\theta=+10^{\circ}$ & no leakage & drainage & & \\
\hline
\end{tabular}

\section{FIGURE CAPTIONS}

Figure 1 - View of a boom.

Figure 2 - The five different modes of oil leakage (from top to bottom):

drainage, entrainment, splash-over, boom submergence and boom planning.

Figure 3 - View of a vertical section of the boom and oil spill. Notation.

Figure 4 - Oil particles leaving the spill to oscillate in the water following large turbulent eddies (experiments carried out at CEDRE).

Figure 5 - LEPTAB's experimental setup (Abed-Meraïm, 2006). 
Figure 6 - Experiments in the LEPTAB channel (Abed-Meraïm, 2006), for $U_{s}=$ $0.7 \mathrm{~m} / \mathrm{s}$ (value in nature) and skirt angle $\theta=+10^{\circ}$. Leakage by entrainment.

Figure 7 - Experiments in the LEPTAB channel (Abed-Meraïm, 2006), for $U_{s}=0.7 \mathrm{~m} / \mathrm{s}$ (value in nature) and skirt angle $\theta=+10^{\circ}$. Leakage by drainage.

Figure 8 - Left: particle interactions are commonly restricted to a finite neighbourhood bounded by a disc in 2D. Right: Wall and ghost particles are used to ensure a Neumann pressure condition on solid boundaries.

Figure 9 - Discretization of the boom in the SPH method.

Figure $10-$ Choice of skirt angles $\theta$ as function of the considered section. Due to the boom curvature in three dimensions, $\theta$ can be either positive or negative.

Figure $11-$ Molecular dynamic viscosity $\left(\right.$ in $\mathrm{m}^{2} \mathrm{~s}^{-1}$ ) of the emulsion as a function of scalar rate-of-strain (in s). Solid circles: measurements conducted by CEDRE; continuous line: model given by equation (37).

Figure 12 - Sketch of the numerical open-channel flow.

Figure 13 - Sketch of the numerical wave flume.

Figure 14 - Numerical open channel flow. Light oil, skirt angle $\theta=+10^{\circ}$ and surface velocity $U_{s}=0.7 \mathrm{~m} / \mathrm{s}$ : different stages of the leakage by entrainment (from top to bottom and left to right). 
Figure 15 - Numerical open channel flow. Heavy oil, skirt angle $\theta=+10^{\circ}$ and surface velocity $U_{s}=0.3 \mathrm{~m} / \mathrm{s}$ : different stages of the leakage by entrainment then drainage (from top to bottom and left to right).

Figure 16 - Entrainment process: comparison between the present SPH computations (left) and LEPTAB's experiments (Abed-Meraïm, 2006), for the heavy oil, $U_{s}=0.3 \mathrm{~m} / \mathrm{s}$ (value in reality) and skirt angle $\theta=+10^{\circ}$.

Figure 17 - Spatial distribution of the maximum value (in time) of the $\sigma$ parameter (defined by equation (17)) for a light oil and skirt angle $\theta=+10^{\circ}$. Top: $U_{s}=0.3 \mathrm{~m} / \mathrm{s}$; bottom: $U_{s}=0.7 \mathrm{~m} / \mathrm{s}$ (horizontal and vertical co-ordinates are in meters).

Figure 18 - Time evolution of the maximum value (in space) of the $\sigma$ parameter (defined by equation (17)) for a light oil and skirt angle $\theta=+10^{\circ}$. Red line: $U_{s}=$ $0.3 \mathrm{~m} / \mathrm{s}$ (no leakage); black line: $U_{s}=0.7 \mathrm{~m} / \mathrm{s}$ (entrainment). The horizontal line corresponds to the critical value $\sigma=5.44$, whereas the vertical one indicates the approximate time when leakage begins for $U_{s}=0.7 \mathrm{~m} / \mathrm{s}$.

Figure 19 - Numerical open channel flow. Effect of the type of oil for a surface velocity $U_{s}=0.7 \mathrm{~m} / \mathrm{s}$ and skirt angle $\theta=+10^{\circ}$. From top to bottom: light oil (weak entrainment), heavy oil (drainage), emulsion (drainage).

Figure 20 - Numerical open channel flow. Effect of the skirt angle $\theta$ for a light oil and surface velocity $U_{s}=0.7 \mathrm{~m} / \mathrm{s} . \theta=+10^{\circ}$ (top) and $\theta=+40^{\circ}$ (bottom). 
Figure 21 - Numerical wave flume. Light oil, skirt angle $\theta=-10^{\circ}$ and wave height $H_{w}=1.0 \mathrm{~m}$ : different stages of the leakage by splash-over (from top to bottom).

Figure $22-$ Numerical wave flume. Heavy oil, skirt angle $\theta=-10^{\circ}$ and wave height $H_{w}=1.0 \mathrm{~m}$ : different stages of the leakage by splash-over (from top to bottom).

Figure 23 - Numerical wave flume. Light oil, skirt angle $\theta=+10^{\circ}$ and wave height $H_{w}=1.0 \mathrm{~m}$ : different stages of the motion without leakage (from top to bottom).

Figure 24 - Numerical wave flume. Effect of the skirt angle $\theta$ for a heavy oil and wave height $H_{w}=1.0 \mathrm{~m}$ : splash-over for $\theta=-10^{\circ}$ (top); drainage for $\theta=+10^{\circ}$ (bottom).

Figure 25 - Numerical wave flume. Effect of the wave height $H_{w}$ with a light oil and skirt angle $\theta=-10^{\circ}$ : no leakage for $H_{w}=0.5 \mathrm{~m}$ (top); splash-over for $H_{w}=$ $1.0 \mathrm{~m}$ (bottom).

Figure 26 - Numerical wave flume. Effect of type of oil for a wave height $H_{w}=$ $1.0 \mathrm{~m}$ and skirt angle $\theta=+10^{\circ}$ : no leakage in the light oil case (top); drainage in the heavy oil case (bottom). 
FIGURES

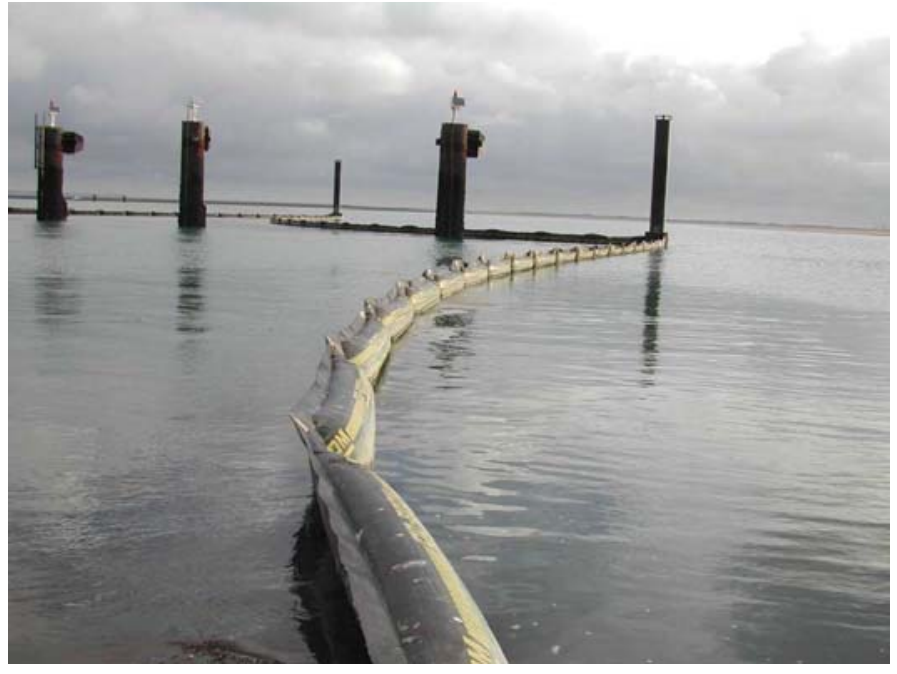

Figure 1 (D. Violeau) 



Figure 2 (D. Violeau) 


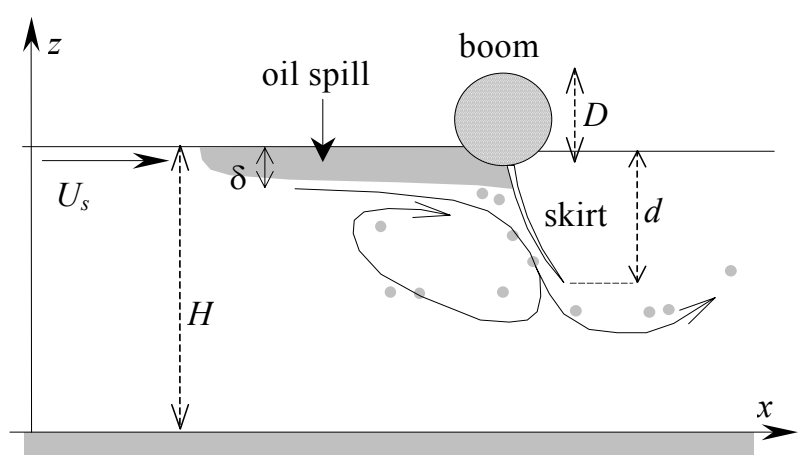

Figure 3 (D. Violeau)

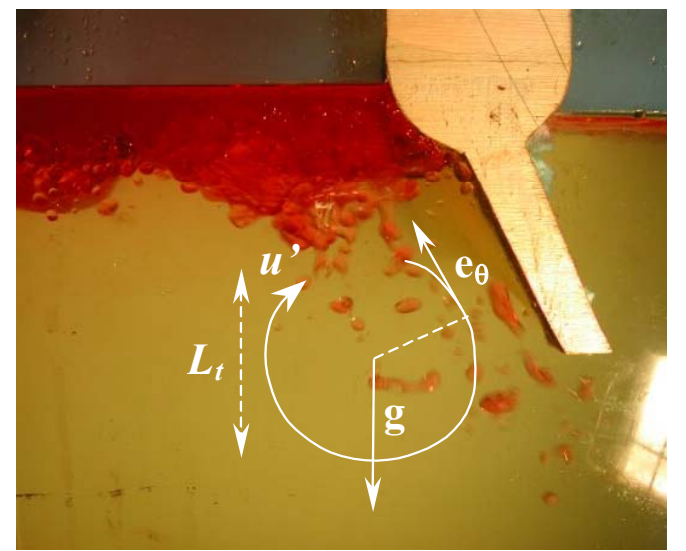

Figure 4 (D. Violeau)

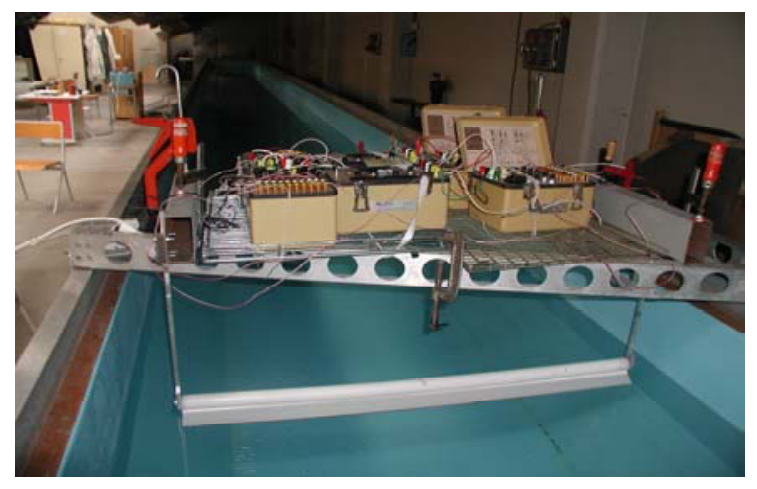

Figure 5 (D. Violeau) 

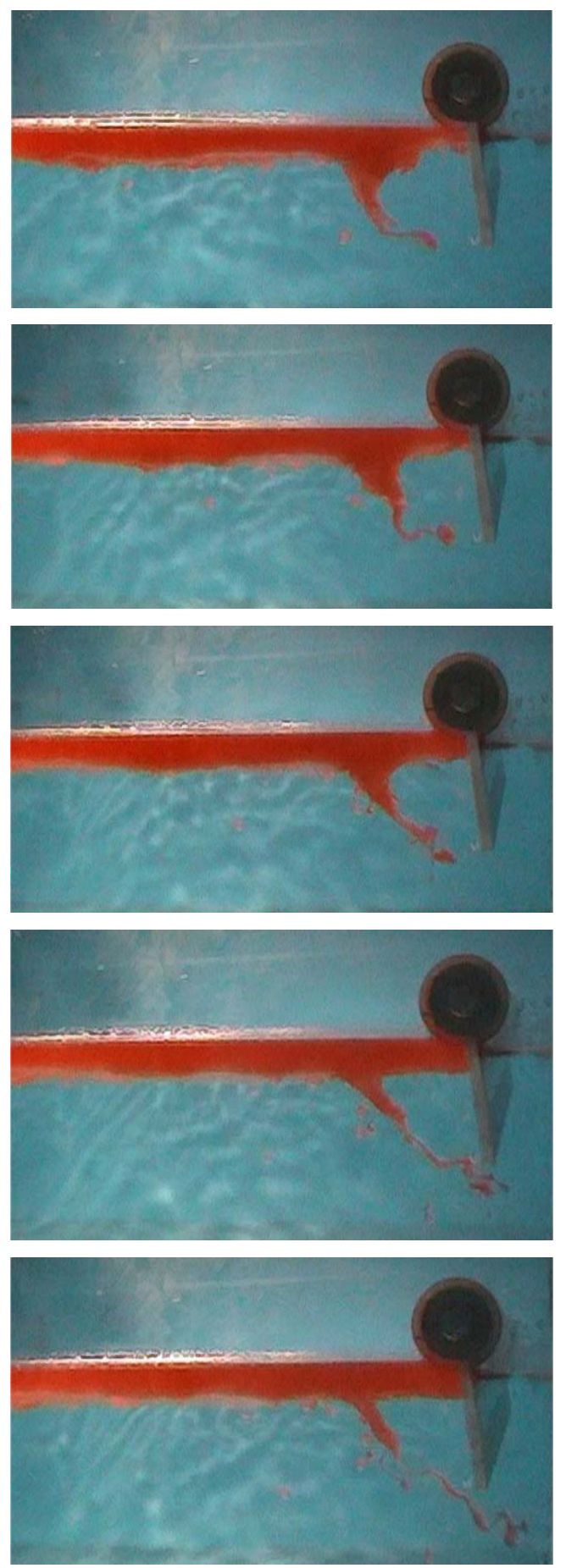

Figure 6 (D. Violeau) 


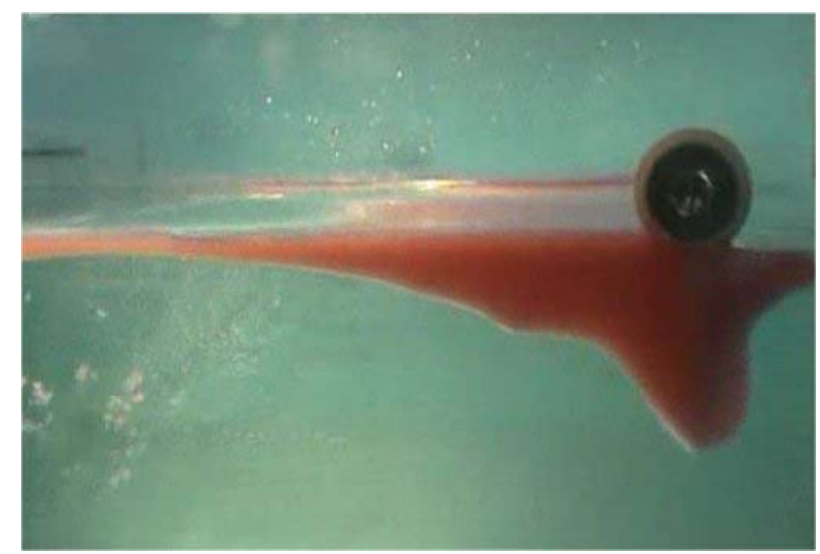

Figure 7 (D. Violeau)
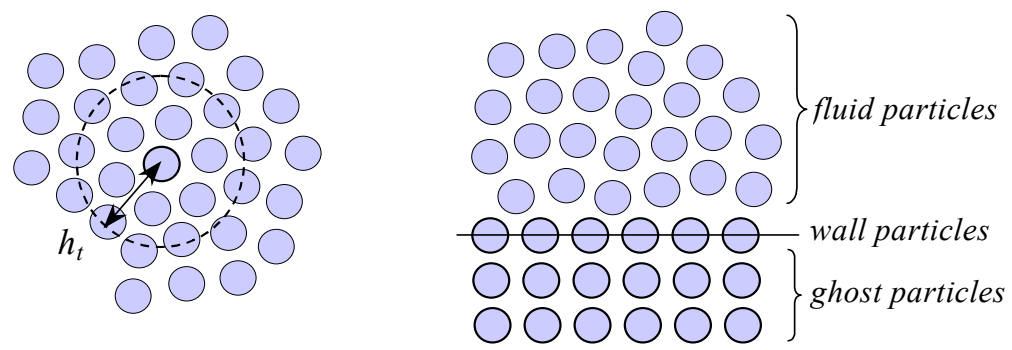

Figure 8 (D. Violeau) 


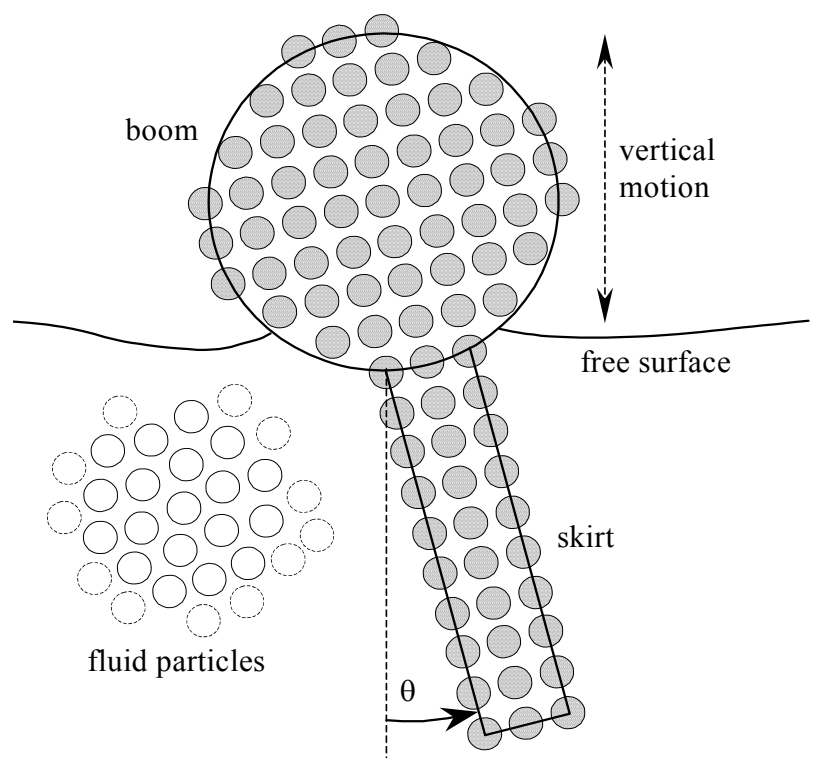

Figure 9 (D. Violeau)

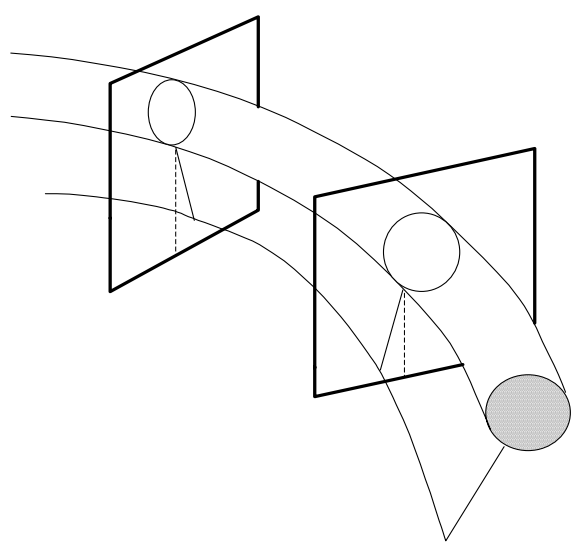

Figure 10 (D. Violeau) 


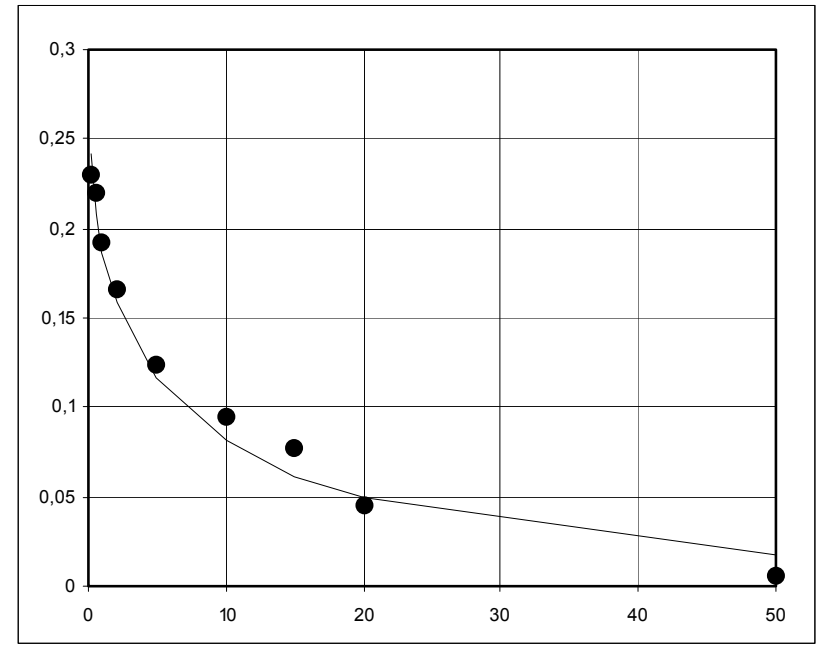

Figure 11 (D. Violeau)

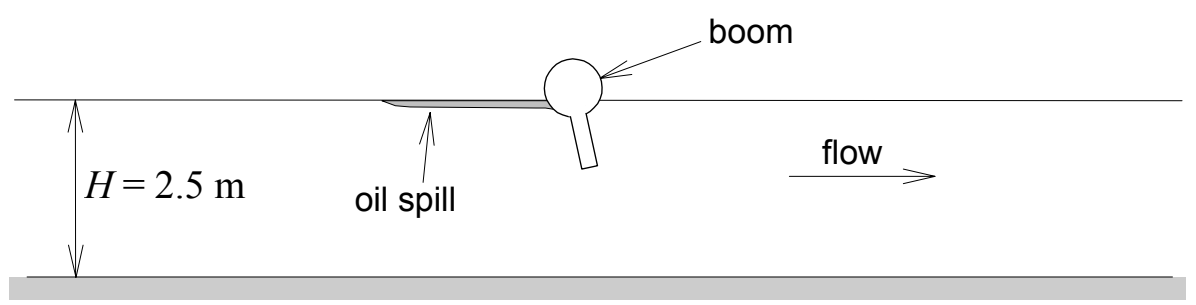

Figure 12 (D. Violeau)

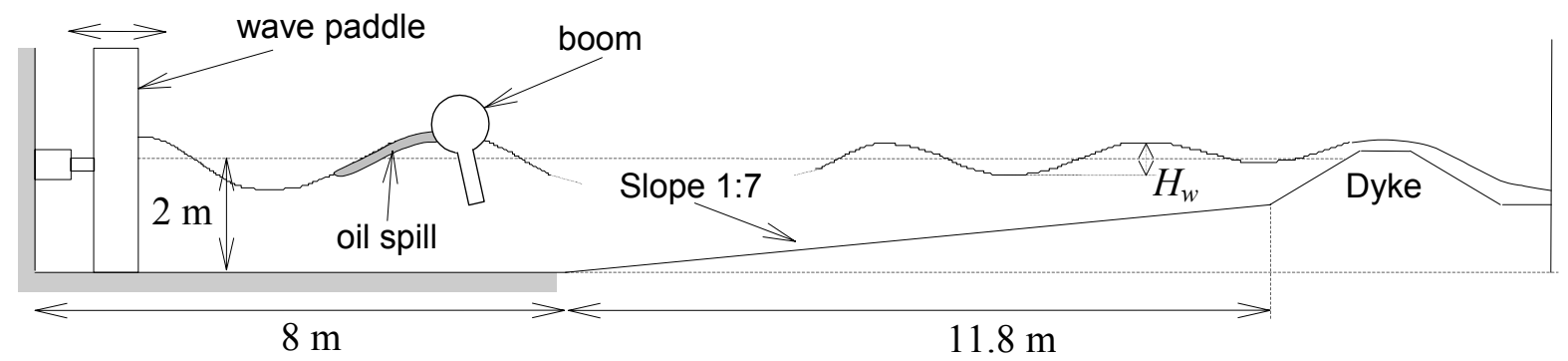

Figure 13 (D. Violeau) 

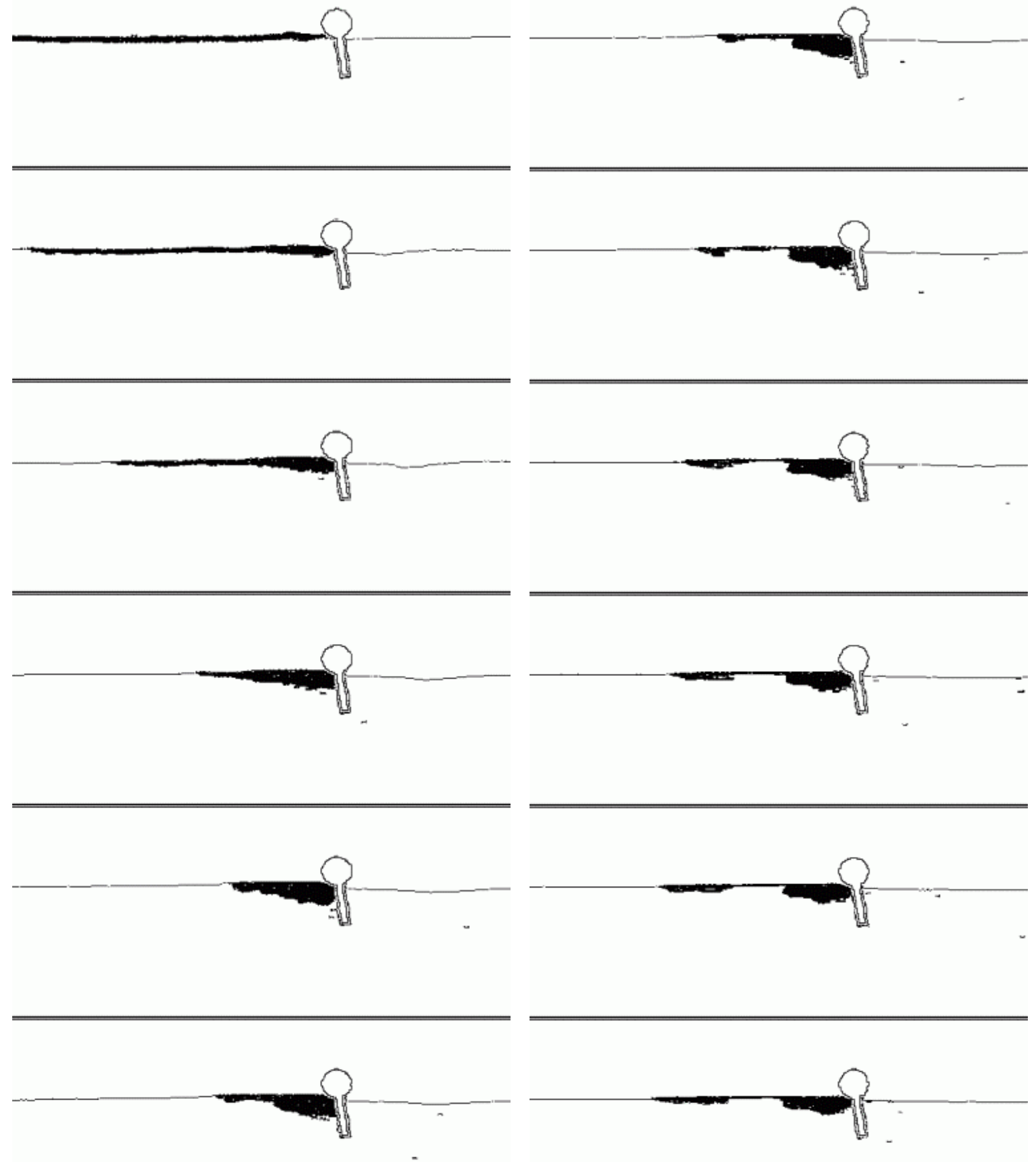

Figure 14 (D. Violeau) 

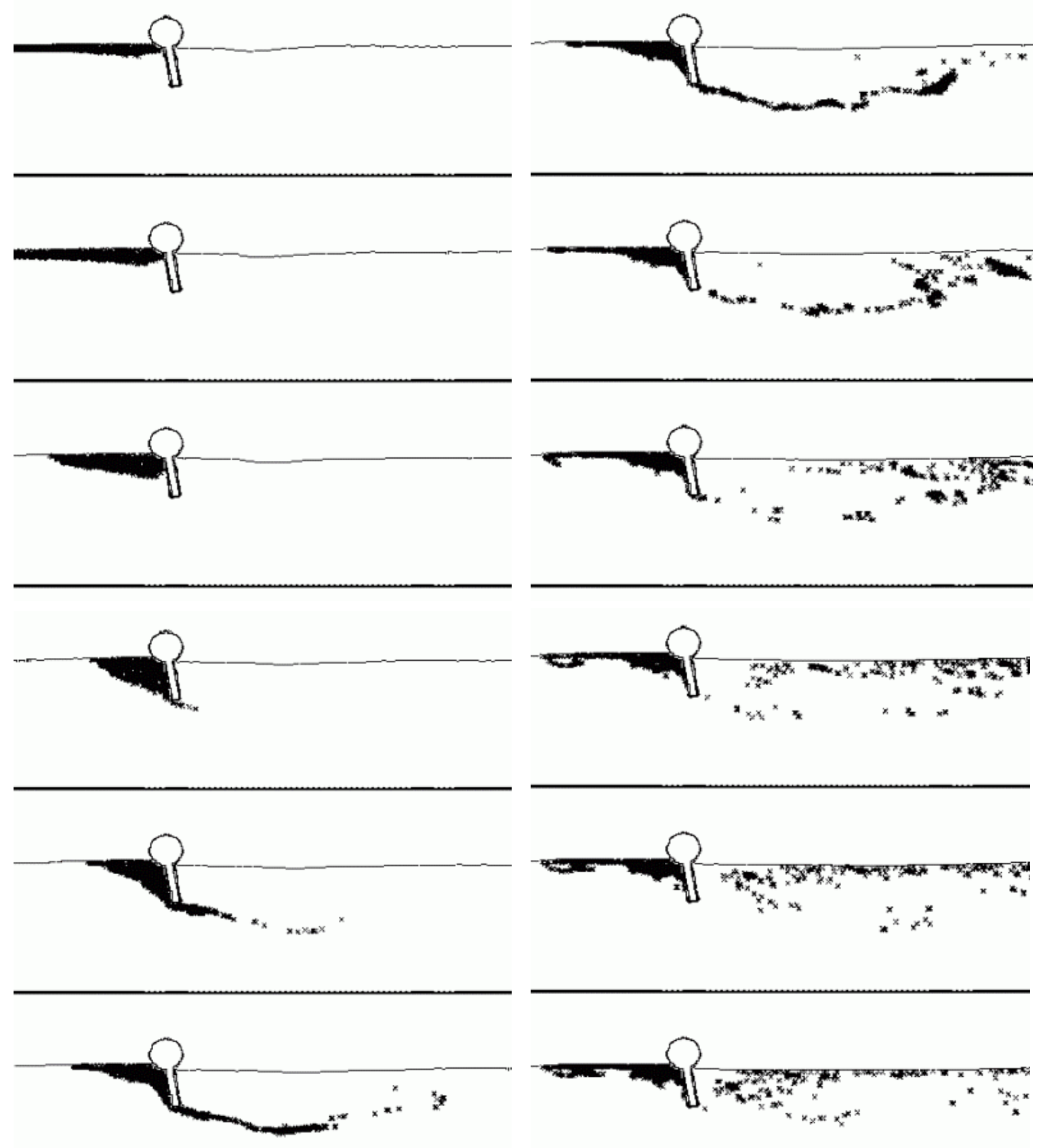

Figure 15 (D. Violeau) 


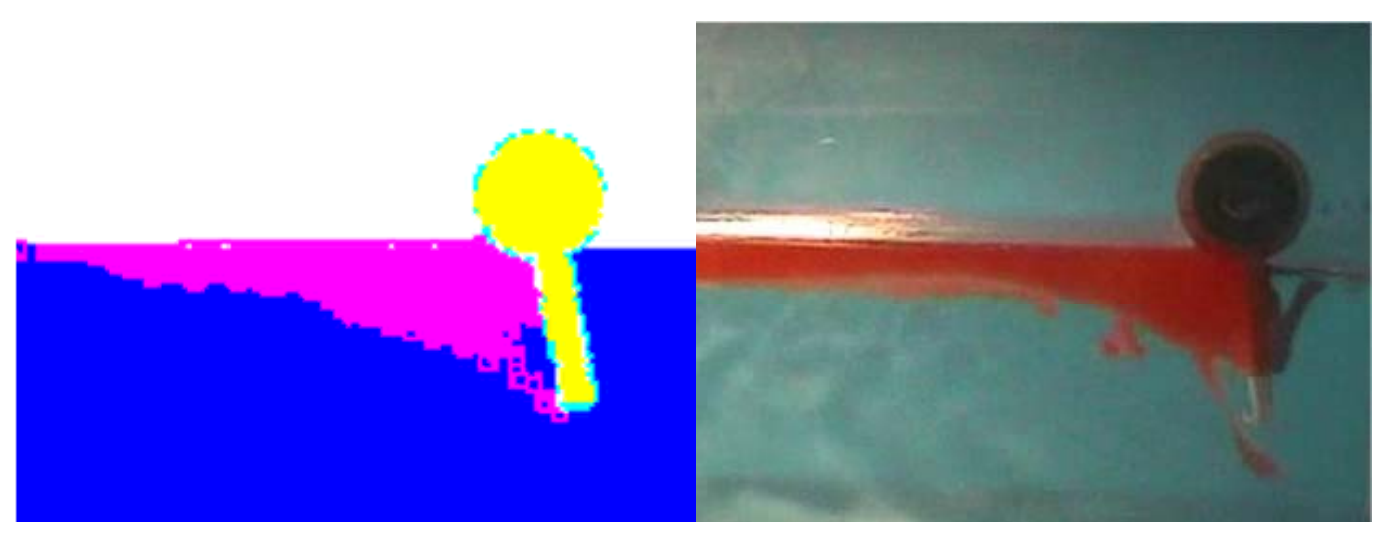

Figure 16 (D. Violeau)

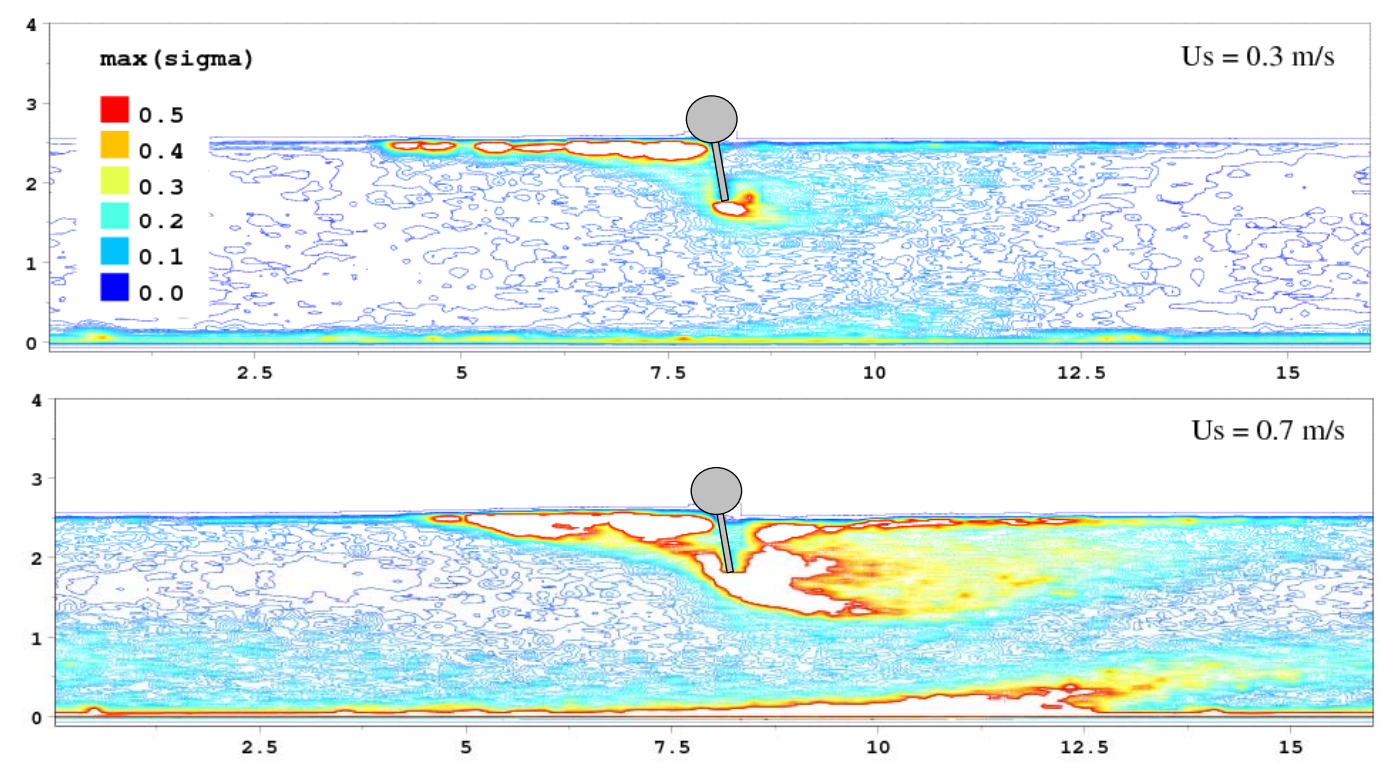

Figure 17 (D. Violeau) 


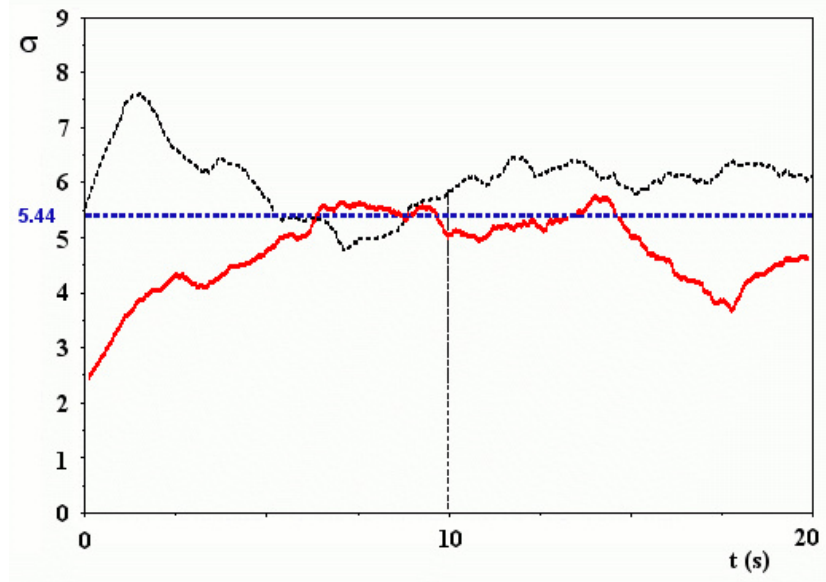

Figure 18 (D. Violeau)
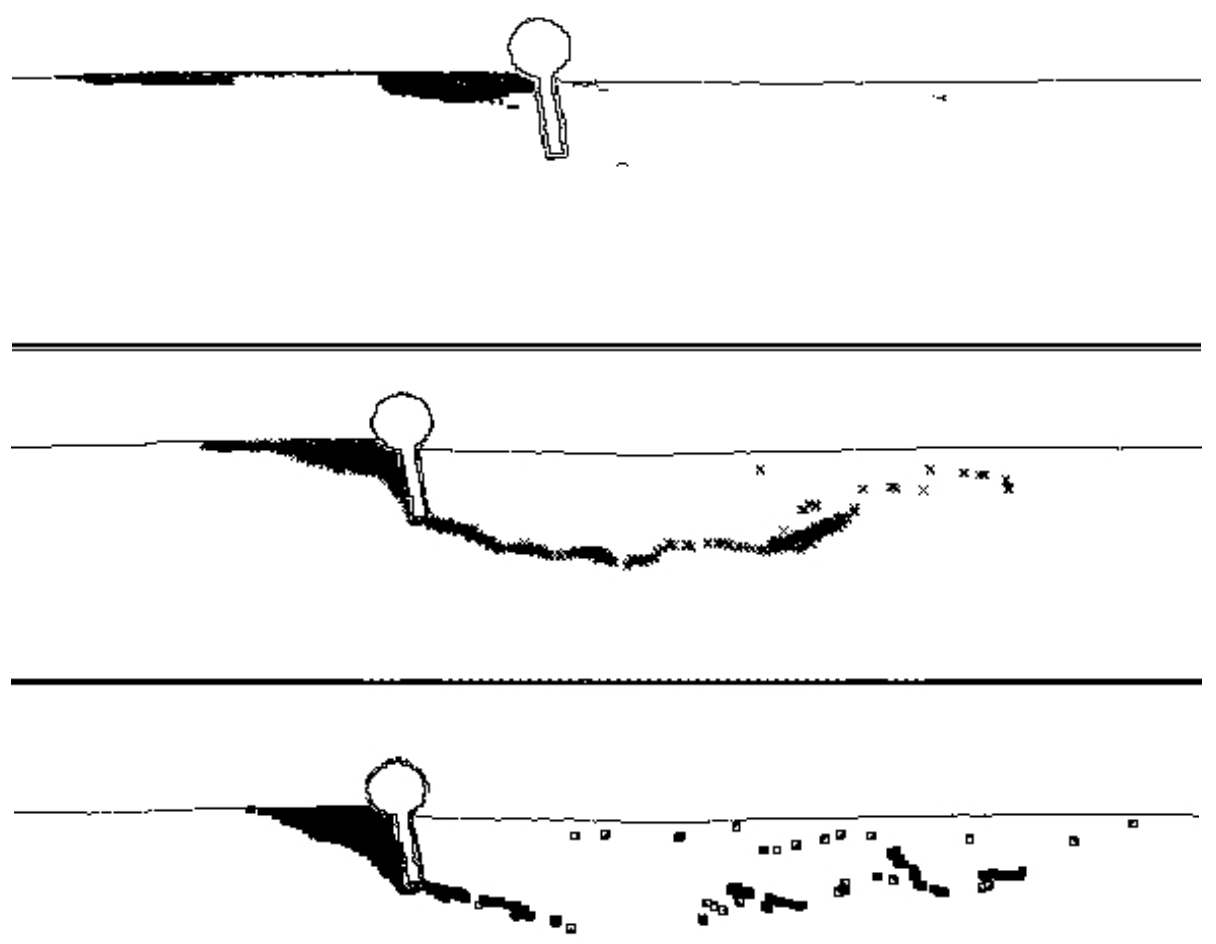

Figure 19 (D. Violeau) 

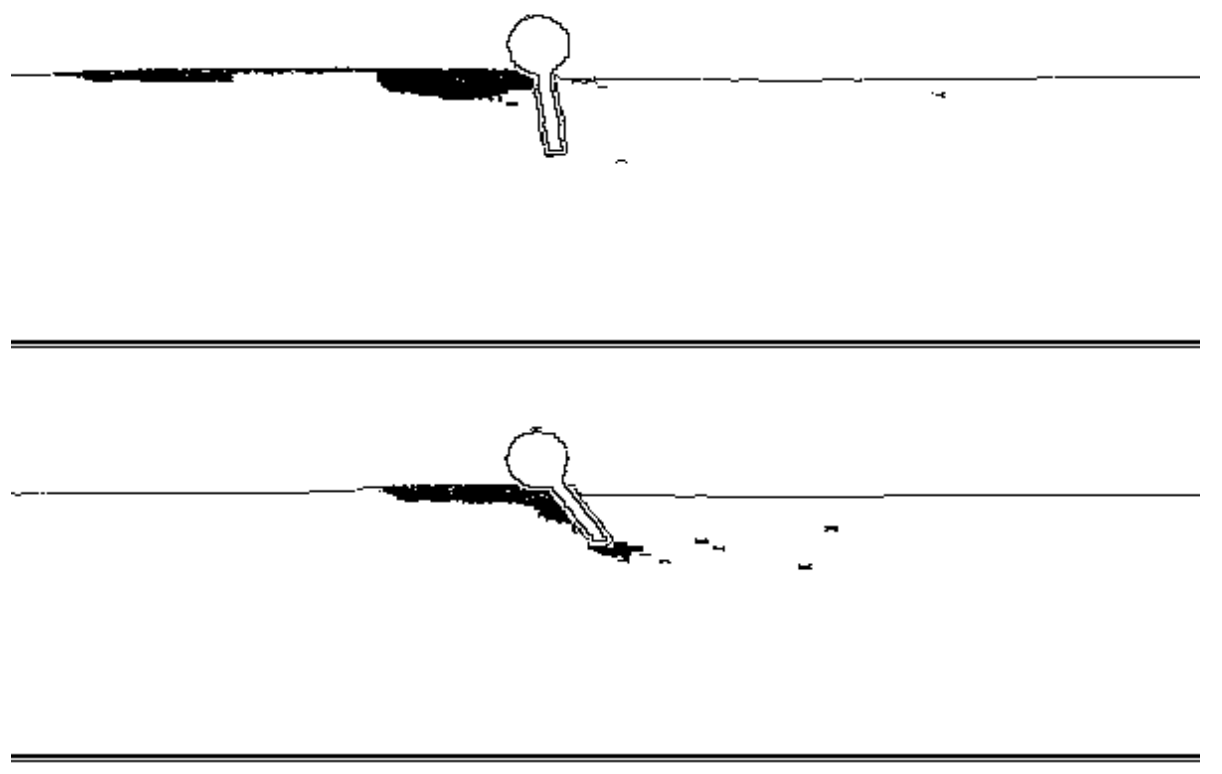

Figure 20 (D. Violeau)
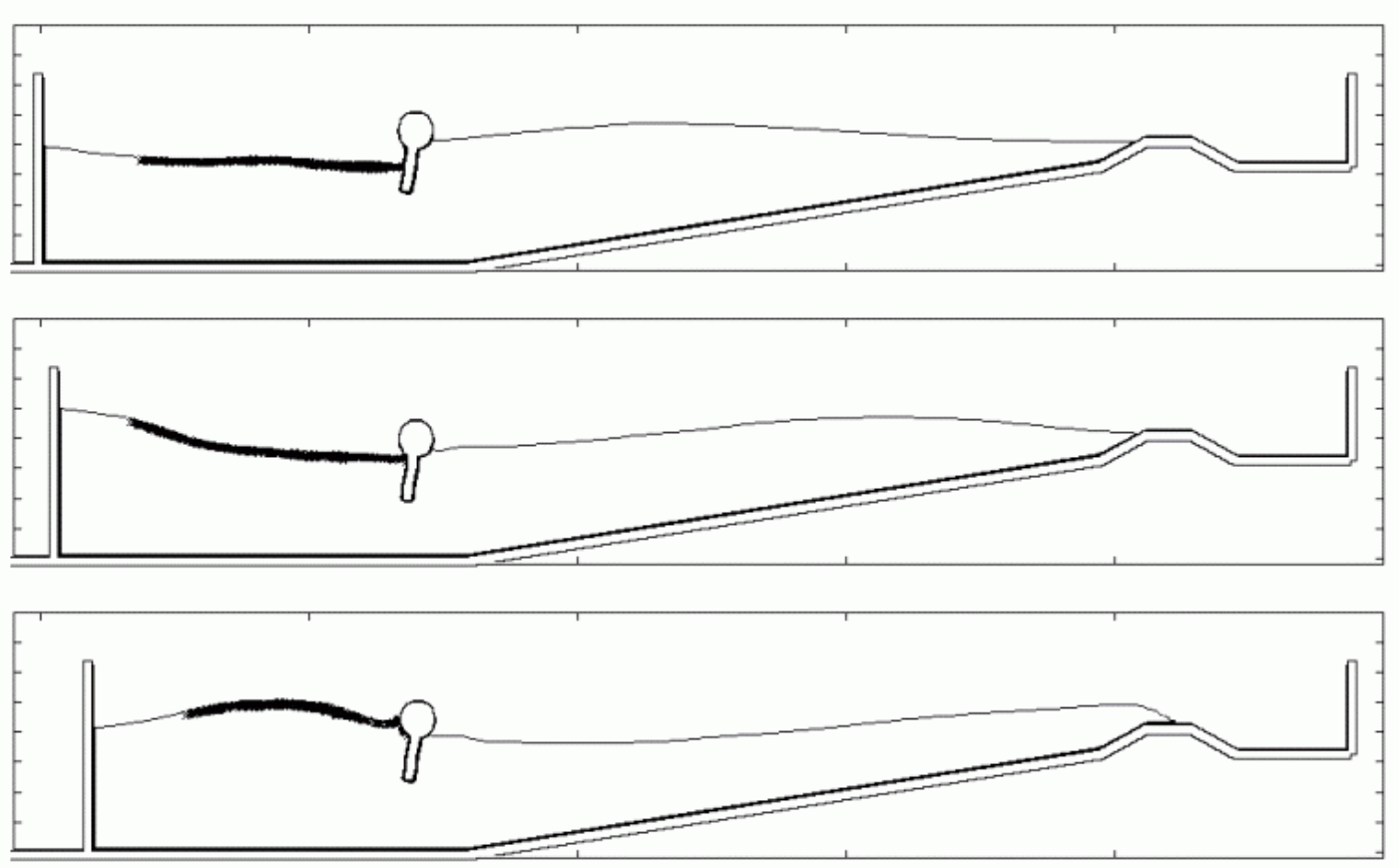

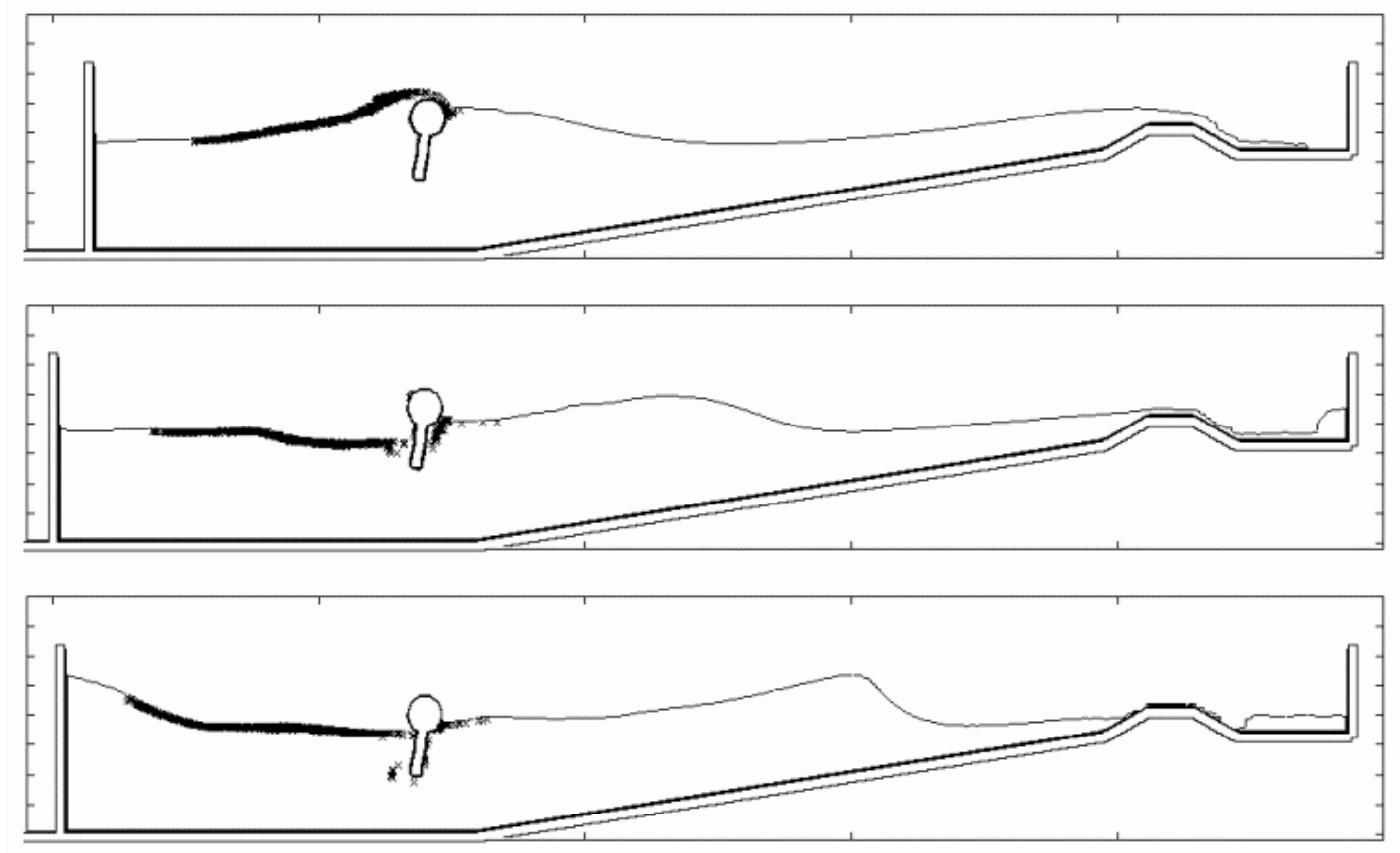

Figure 21 (D. Violeau) 

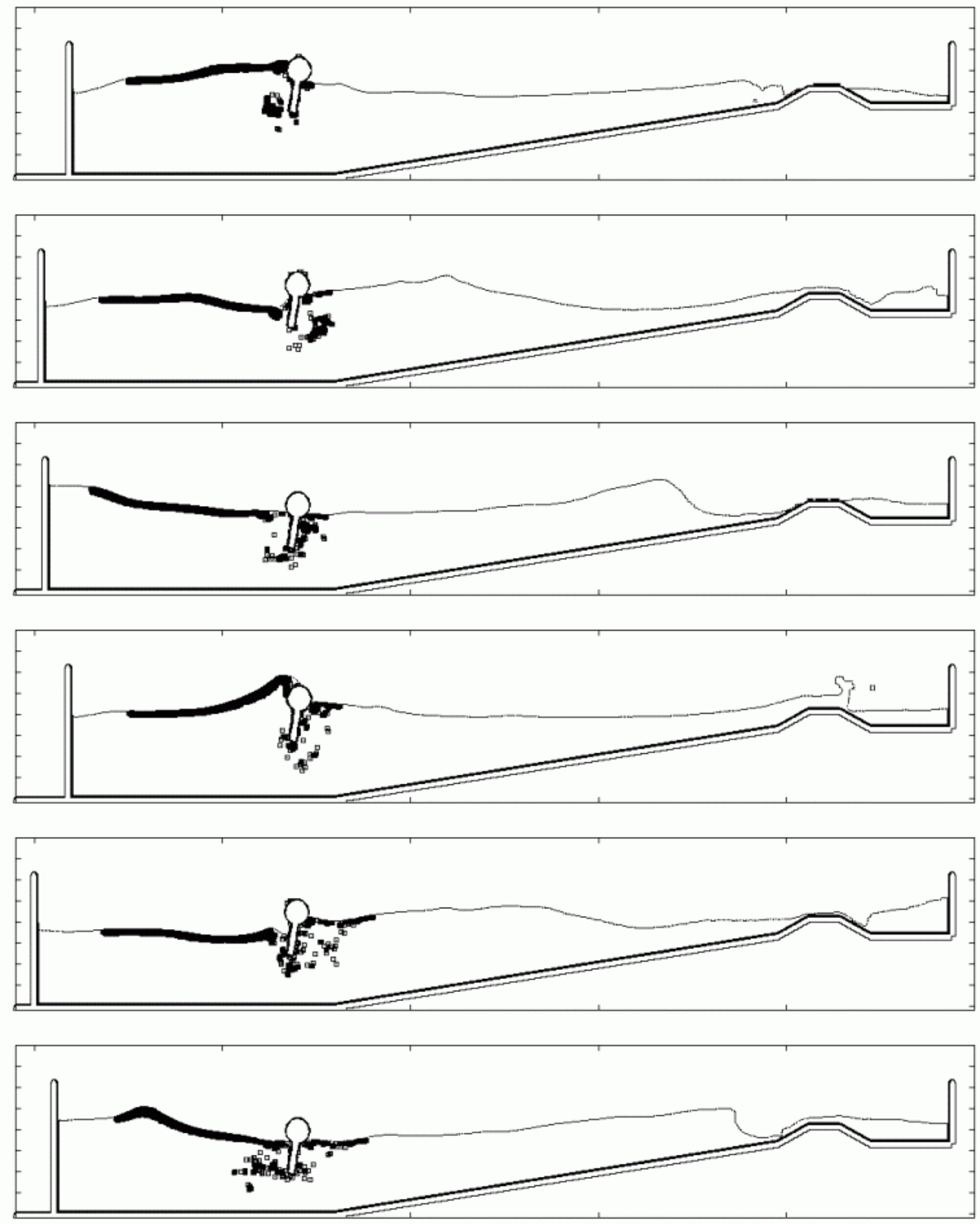

Figure 22 (D. Violeau) 

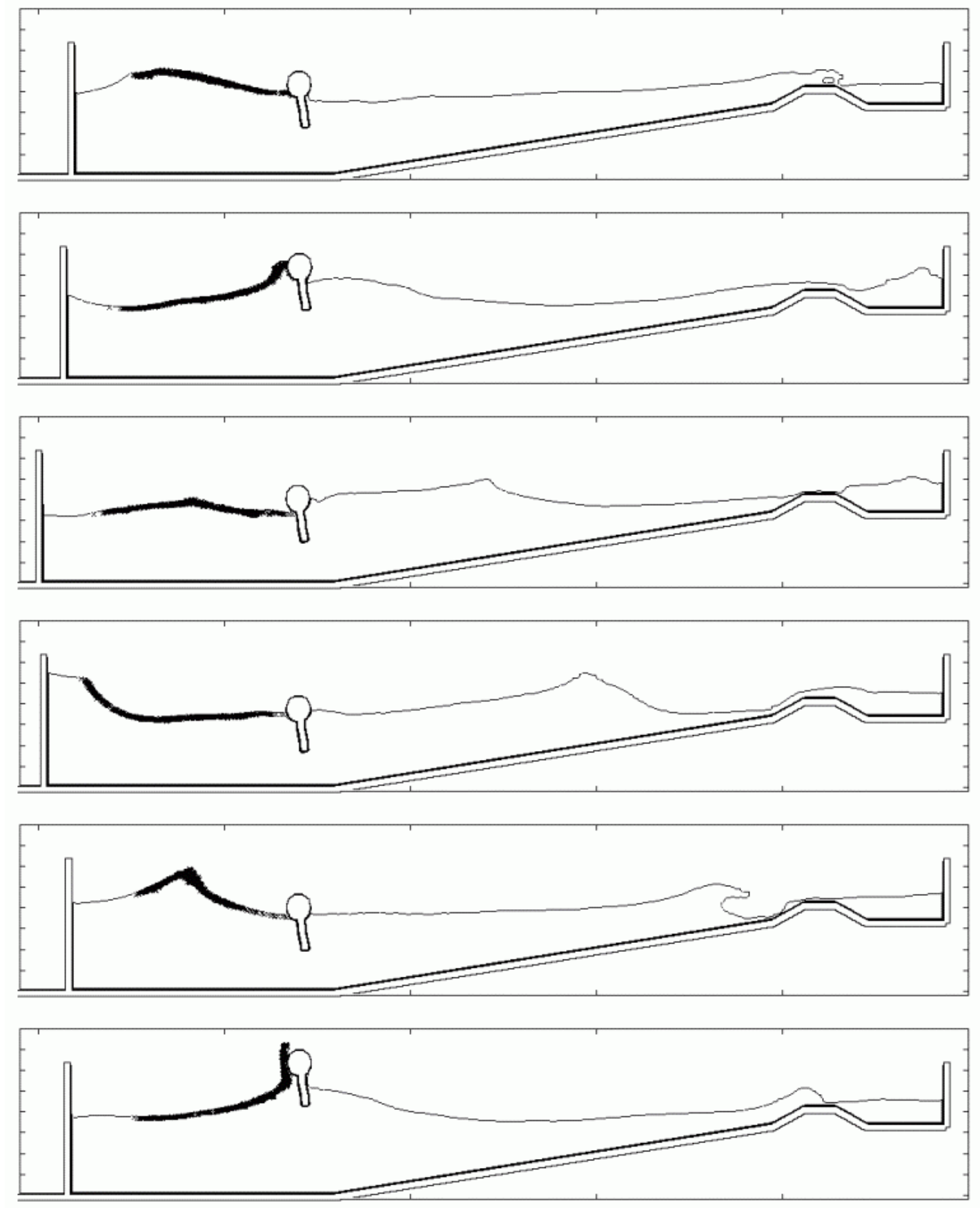

Figure 23 (D. Violeau) 

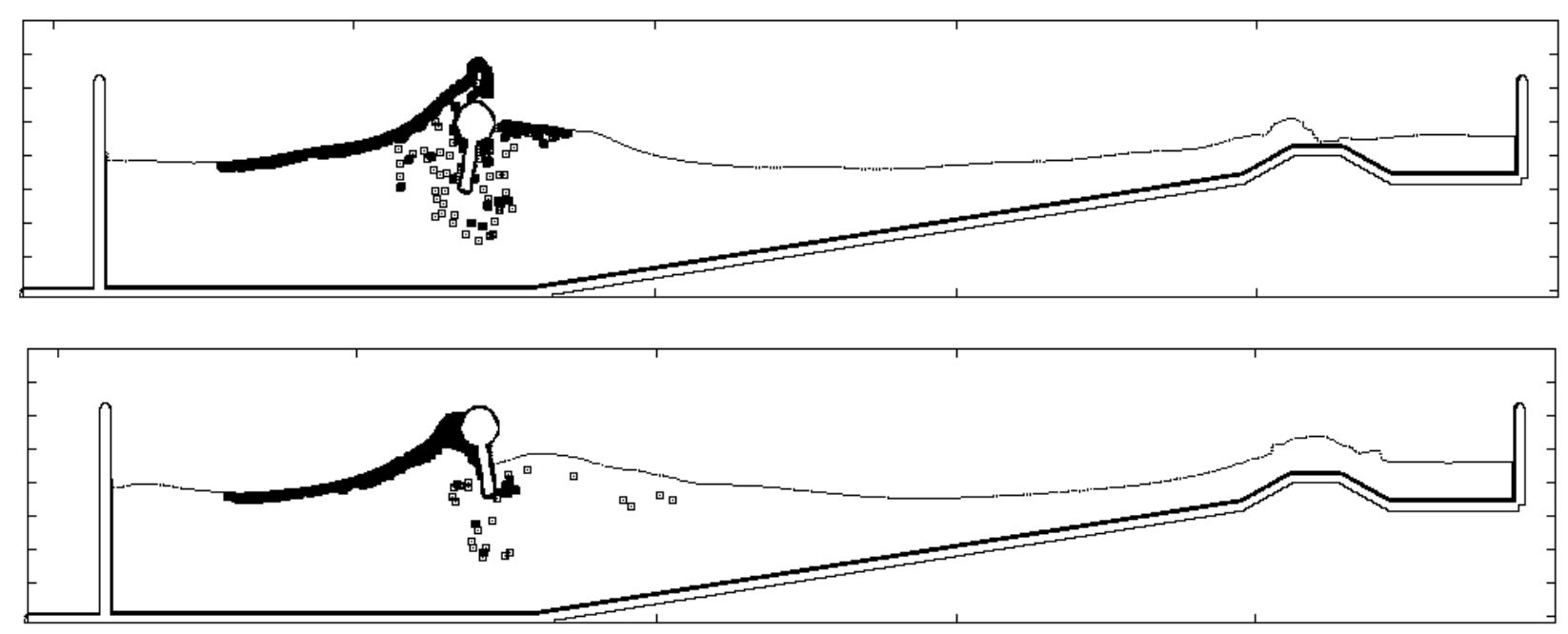

Figure 24 (D. Violeau)
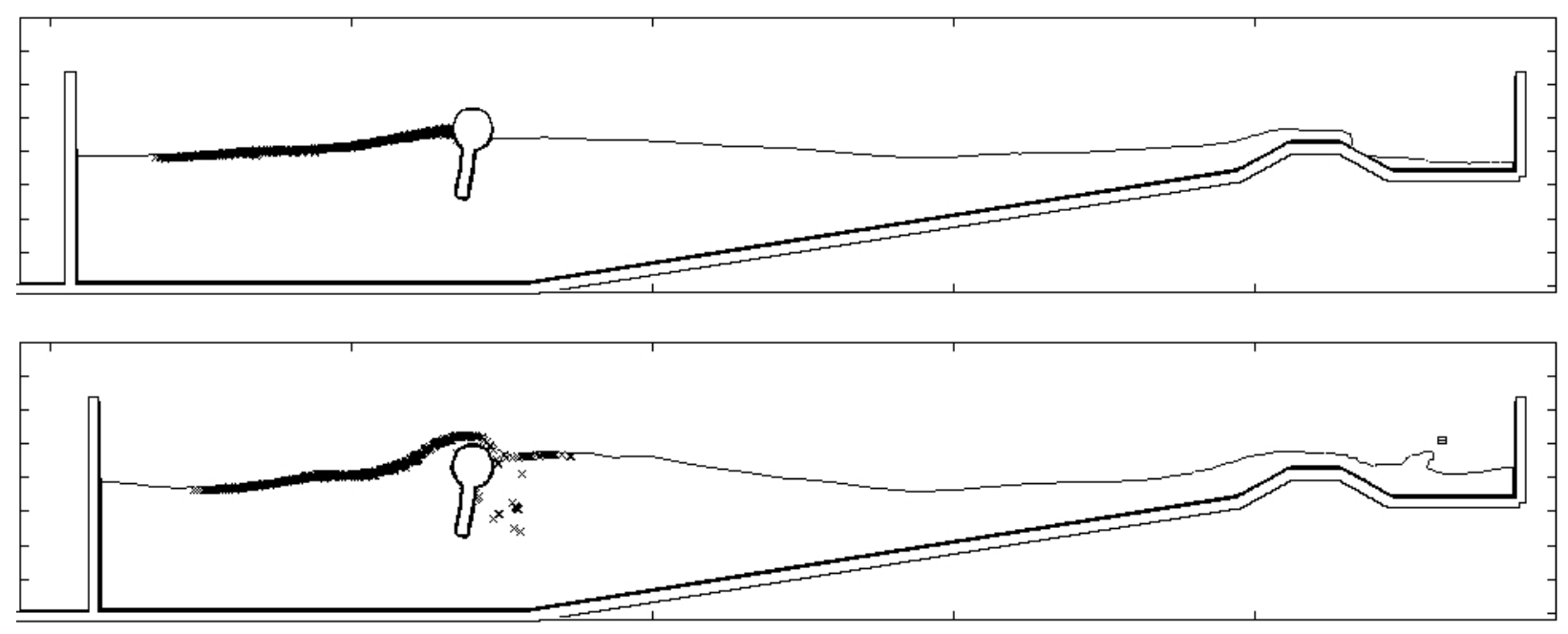

Figure 25 (D. Violeau)

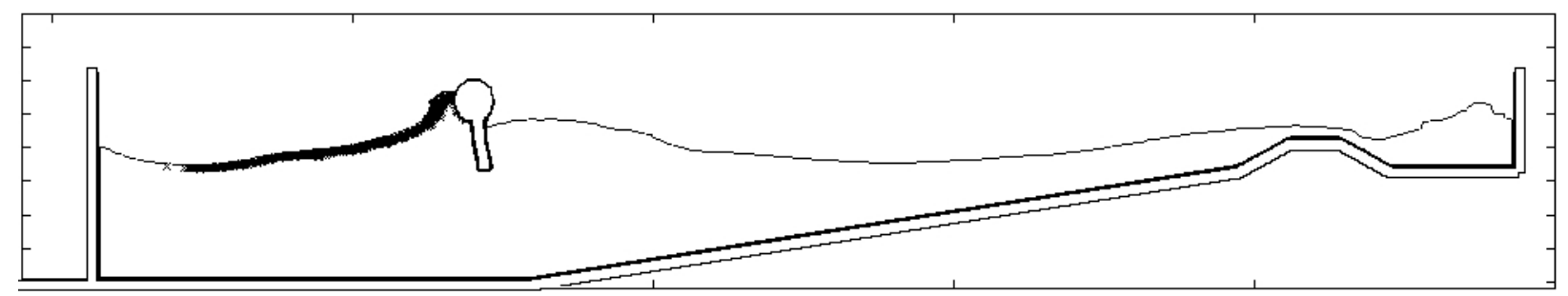




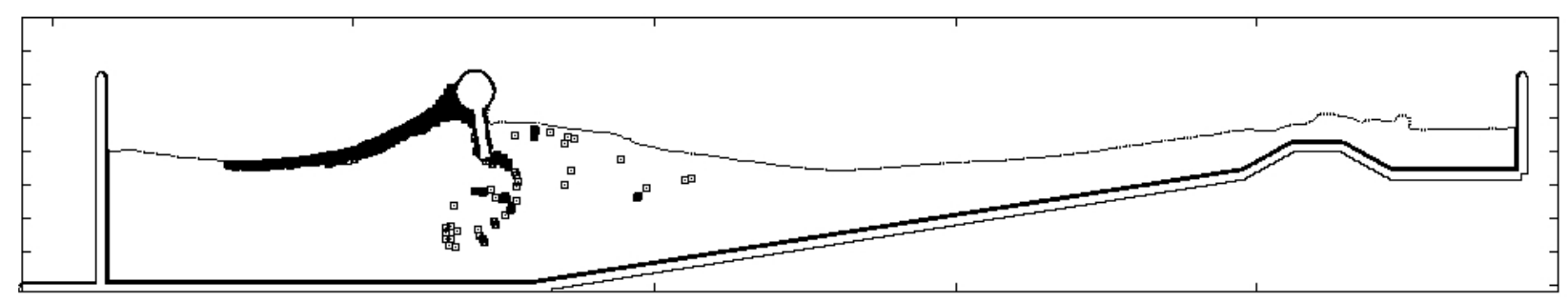

Figure 26 (D. Violeau) 